\title{
Nano-Thermal Transport Array: An Instrument for Combinatorial Measurements of Heat Transfer in Nanoscale Films
}

\section{Citation}

McCluskey, Patrick James, and Joost J. Vlassak. Forthcoming. Nano-thermal transport array: An instrument for combinatorial measurements of heat transfer in nanoscale films. Thin Solid Films 518.

\section{Published Version}

doi:10.1016/j.tsf.2010.05.124

\section{Permanent link}

http://nrs.harvard.edu/urn-3:HUL.InstRepos:4317715

\section{Terms of Use}

This article was downloaded from Harvard University's DASH repository, and is made available under the terms and conditions applicable to Open Access Policy Articles, as set forth at http:// nrs.harvard.edu/urn-3:HUL.InstRepos:dash.current.terms-of-use\#OAP

\section{Share Your Story}

The Harvard community has made this article openly available.

Please share how this access benefits you. Submit a story.

\section{Accessibility}




\title{
nano-Thermal Transport Array: An Instrument for Combinatorial Measurements of Heat Transfer in Nanoscale Films
}

\author{
Patrick J. McCluskey, Joost J. Vlassak* \\ School of Engineering and Applied Sciences, Harvard University \\ Cambridge, MA 02138, United States
}

\begin{abstract}
The nano-Thermal Transport Array is a silicon-based micromachined device for measuring the thermal properties of nanoscale materials in a high-throughput methodology. The device contains an array of thermal sensors, each one of which consists of a silicon nitride membrane and a tungsten heating element that also serves as a temperature gauge. The thermal behavior of the sensors is described with an analytical model. The assumptions underlying this model and its accuracy are checked using the finite element method. The analytical model is used in a data reduction scheme that relates experimental quantities to materials properties. Measured properties include thermal effusivity, thermal conductivity, and heat capacity. While the array is specifically designed for combinatorial analysis, here we demonstrate the capabilities of the device with a high-throughput study of copper multi-layer films as a function of film thickness, ranging from 15 to $470 \mathrm{~nm}$. Thermal conductivity results show good agreement with earlier models predicting the conductivity based on electron scattering at interfaces.
\end{abstract}

\section{Introduction}

The thermal transport properties of nanoscale materials can differ significantly from their bulk counterparts $[1,2]$. When the microstructural length scale of a material is comparable to the mean free path of the phonons and electrons responsible for thermal transport in that material, surfaces and interfaces start to influence overall thermal transport. This effect has become increasingly important, as nanotechnology has pushed device design below the applicable scales of bulk materials properties. In devices such as integrated circuits or micro-electro-mechanical systems, thermal transport can play a significant role in function and failure. Beyond technological considerations, the study of thermal properties at the nano-scale presents interesting fundamental questions into the

* Corresponding author. Email address: vlassak@esag.harvard.edu 
interaction of heat transfer and microstructure at these small length scales, including thermal conductivity across and parallel to interfaces [3].

Along with length scale, thermal properties of materials also vary with composition and processing conditions [4]. Extensive studies of these considerations have been performed for bulk materials in the past, but few studies have been performed on nanoscale materials systems. A high-throughput measurement technique specifically applicable to nanoscale materials would be helpful in remedying this situation. Such a technique could be used to quickly map the thermal transport properties of very small quantities of materials as a function composition or processing conditions, thus facilitating design and optimization of nanoscale devices.

Thin-film heat transport metrology has improved significantly in recent years. Through-plane techniques such as 3-omega, time-domain thermoreflectance, and scanning optical thermometry can be used in a high-throughput methodology, but have limited in-plane applicability [5,6]. The most promising in-plane methods rely on membrane structures that constrain heat flow in the plane [7-9]. Until now, this class of instruments has seen no high-throughput thermal conductivity measurement application. Many of the membrane-based techniques are not compatible with high-throughput methods because they involve multiple thermal cells and/or numerous thermometers. The 3-omega method has been used to measure in-plane thermal conductivity, but the technique requires two heating elements of differing widths for each measurement, reducing the high-throughput applicability of this approach [10]. More recently the 3omega method has been used to measure the thermal conductivity and heat capacity of silicon nitride and nickel titanium membranes. The approach used for these measurements requires multiple thermometers and up to 16 electrical connections, making it inconvenient for high-throughput application [11].

In the following article, we present an instrument designed to measure the inplane thermal conductivity of nanoscale films and coatings with a high-throughput 
methodology. The nano-thermal transport array (nTTA) is a micromachined array of thermal sensors, so called because it uses a high-throughput approach to measuring heat transport in thin film material systems. An analytical, one-dimensional, transient heat transfer model is used to optimize the dimensions of each sensor, define the measurement range, and reduce measured data to the desired material properties. A finite element model (FEM) is used to verify the analytical model. Direct effusivity measurements of $\mathrm{Si}_{3} \mathrm{~N}_{4}$ membranes and $\mathrm{Si}_{3} \mathrm{~N}_{4} / \mathrm{Cu}$ multilayer-membranes as a function of film thickness, and calculated thermal conductivity measurements, demonstrate the capabilities of the nTTA.

\section{Physical Description and Operating Principles}

The nTTA device consists of a substrate with a number of micromachined thermal sensors. The thermal sensors are arranged in a $5 \times 5$ array to facilitate combinatorial sample preparation (Fig. 1). When a thin-film sample with an in-plane composition or thickness gradient is deposited on this substrate, it is essentially discretized at each thermal sensor allowing the simultaneous measurement of 25 samples with unique composition or thickness.

The design and operation of the thermal sensors is similar to the nano-calorimetric cells developed by Allen and colleagues [12-15], but optimized for sensitivity to heat loss. Each thermal sensor consists of a thermistor on an electrically insulating ceramic membrane supported by the substrate (Fig. 2(a), (b)). The thermistor is fabricated from an electrically conductive film and serves both for measuring temperature and for heating the sample. Samples to be measured form a continuous film across the membrane of each sensor. Electrically insulating samples are deposited on the front-side of the membrane while conductive samples are deposited on the backside of the membrane.

Referring to the schematic in Fig. 2(a), the long straight line down the center of the membrane is the heating element, the metal lines connected to the heater are the 
voltage probes, and the portion of the heating element between the voltage probes is the thermistor. A current passed through the heating element heats the membrane along its centerline. The local temperature change is determined from the resistance of the thermistor, which is evaluated in a four-point measurement. As the thermistor heats, a temperature gradient develops in the membrane resulting in heat loss. The thermal properties of the membrane are determined from the power input and temperature history of the thermistor before a steady state is reached, i.e., they are evaluated from the transient behavior of the sensor. As will be discussed later, this approach has some benefit over techniques relying on the steady state in terms of improved temperature uniformity within the sensor.

Conservation of energy relates the electrical power dissipated in the thermistor to the thermal energy stored locally and the heat lost to the environment. The power dissipated is determined experimentally from the current supplied to the thermistor and the potential drop between the voltage probes. The current and the potential drop also provide the resistance of the thermistor, which is calibrated to temperature. The temperature history of the thermistor, thermal properties of the materials, and the geometry of the thermal sensor determine the energy stored and lost. The sensor array contains both sensors with narrow and wide thermistors. At moderate temperatures the heat loss from narrow thermistors is dominated by heat transfer from the thermistor to the membrane, and as a result the temperature response of the thermistor is a strong function of the thermal transport properties of the membrane. Thermal modeling is used to relate power and temperature to the specific heat and effusivity of the membrane. Wide thermistors (rightmost column of thermistors in Fig. 1) are paired with the neighboring narrow thermistors in a measurement scheme to decouple radiation and conduction losses and to facilitate emissivity measurements. Vacuum conditions eliminate convection losses and provide a chemically inert testing environment.

Two measurements are required to extract the thermal properties of the sample film: One reference measurement on the bare thermal cell and one on the cell with the sample film. The reference measurement characterizes the heat loss through the bare 
membrane, while the cell with sample film provides effective thermal properties for the ceramic/sample bilayer membrane. Combining the results of both measurements allows determination of the sample thermal properties. A wide range of samples can be deposited and measured as long as they do not fracture the membranes due to high stresses.

Materials chosen for the nTTA device are based on the functional requirements placed on each structure, cost and fabrication knowledge base. The substrate is made of silicon, chosen because of the availability of relatively low-cost and high-quality wafers. Silicon also has established anisotropic etching procedures for forming cavities. The ceramic membrane is made of $\mathrm{Si}_{3} \mathrm{~N}_{4}$, selected because of its electrically insulating properties and a demonstrated ability to form thin membranes. The thermistor is made of tungsten, because of its relatively large temperature coefficient of resistance and its small resistivity, which are beneficial to measurement sensitivity [13]. Also, tungsten has a very high melting point, so that the material has excellent thermal stability compared to conductors with lower melting temperatures. The electrical leads and contact pads on the substrate are made of copper to reduce the resistance of the lead lines and to facilitate contact to the device. The high-temperature stability of nTTA materials means that the device is compatible with high-temperature sample fabrication methods. Specific dimensions of the nTTA device can be found in Table 1.

\section{Transient 2D Thermal Model}

This section is focused on the derivation of the thermal model that describes the sensor. Much of the detail of the modeling consists of formulating the heat flow in the membrane. To accomplish this task we consider a control volume $\mathrm{CV}$. The $\mathrm{CV}$ is defined by the volume of the thermistor and the volume of the membrane (including any sample) directly below the thermistor (Fig. 3). The CV is bounded by the two $x z$-planes located at the edges of the heating element, the two $y z$-planes at the voltage probes, and the two $x y$ planes at the top and bottom free surfaces of the thermal cell. 
The electrical power dissipated in the thermistor, $P$, is partly stored as internal energy in the CV $U$, and partly lost to the environment by conduction and radiation, $Q$ :

$$
P=U+Q .
$$

The energy storage rate is

$$
U=\iiint_{C V} \rho c_{P} \frac{\partial T}{\partial t} d V,
$$

where $\rho$ is the density, $c_{P}$ is the specific heat, $T$ is the temperature, $t$ is time and $V$ is the volume. The total energy loss to the environment $Q$, is decomposed as,

$$
\begin{aligned}
& Q=Q_{x}+Q_{y}+Q_{z}, \\
& Q_{x}=\iint-2 k_{x} \frac{\partial T}{\partial x} d A_{x}, \\
& Q_{y}=\iint-2 k_{y} \frac{\partial T}{\partial y} d A_{y}, \\
& Q_{z}=\iint 2 \varepsilon_{C V} \sigma\left(T^{4}-T_{0}^{4}\right) d A_{z},
\end{aligned}
$$

where $x, y$, and $z$ represent the respective directions of the heat flow. Physically, $Q_{x}$ represents the conduction losses at the ends of the thermistor, $Q_{y}$ refers to the conduction losses into the membrane, and $Q_{z}$ represents the radiation losses from the top and bottom free surface of the $\mathrm{CV}$. Within the definition of these terms, $k$ is the thermal conductivity, $\varepsilon$ is the emissivity, $\sigma$ is the Stefan-Boltzmann constant, $T_{0}$ is the temperature of the environment, and $A$ is the respective cross-sectional area. The factor of two in each term arises from the symmetry of the $\mathrm{CV}$. Convection losses are neglected because the measurements are performed in vacuum.

The relations expressed in Eqs. (2) and (3) can be simplified significantly. Since the physical length scale in the $z$-direction is much smaller than a typical diffusive length scale, the temperature is uniform in this direction. Conversely, in the $x$-direction the thermal diffusion length in a typical experiment is much smaller than the distance from the voltage probes to the substrate. Therefore, significant temperature gradients are constrained to the inactive portions of the heater; the temperature of the active portion of 
the heater (i.e. the thermistor) can be considered uniform in the $x$-direction. This transient temperature profile is in contrast to steady-state hot strip techniques, where temperature variation along the heater can be significant. Finally, we assume that temperature variation in the $y$-direction is small within the $\mathrm{CV}$, and confirm this with finite element modeling later in the paper. From these assumptions, the $Q_{x}$ term vanishes while the energy storage rate and the radiation loss reduce to $U=C_{P} d f[t] / d t$ and $Q_{z}=2 A_{z} \varepsilon_{C V} \sigma\left(\left(f[t]+T_{0}\right)^{4}-T_{0}^{4}\right)$, respectively. Here $C_{P}$ is the total heat capacity of the $\mathrm{CV}$ (including sample when applicable) and $f[t]$ represents the average temperature change of the heater, $f[t]=T_{A v e}-T_{0}$, as a function of time. Finally, since $Q_{y}$ must be continuous across the $\mathrm{CV}$-membrane boundary, this term can be rewritten as $Q_{y}=-2 A_{M} k_{M} \partial T_{M} / \partial y$, where the subscript $M$ denotes membrane parameters and the membrane temperature gradient is evaluated at the edge of the heating element. Substituting the expressions for $U, Q_{\mathrm{y}}$, and $Q_{z}$ into Eq. (1) results in

$$
P=C_{P} r[t]-2 A_{M} k_{M} \frac{\partial T_{M}}{\partial y}+2 A_{z} \varepsilon_{C V} \sigma\left(\left(f[t]+T_{0}\right)^{4}-T_{0}^{4}\right) .
$$

Here $r[t]$ is the heating rate of the $\mathrm{CV}, A_{M}$ is the cross-sectional area of the membrane, $k_{M}$ is the thermal conductivity of the membrane, $A_{z}$ is the emitting surface area of the $\mathrm{CV}$, and $\varepsilon_{C V}$ is the effective emissivity of the $\mathrm{CV}$. The emissivity $\varepsilon_{C V}$ is an effective value because the top and bottom surfaces of the CV are made of different materials with different emissivity values. Equation (4) is not yet explicit in terms of the relevant thermal parameters because the temperature gradient in the membrane cannot be measured directly and it depends on both $k_{M}$ and the volumetric heat capacity of the membrane $\left(\rho c_{P}\right)_{M}$.

To determine the temperature gradient in the membrane, we solve the onedimensional thermal diffusion equation for the temperature profile in the membrane,

$$
\left(\rho c_{P}\right)_{M} \frac{\partial T_{M}}{\partial t}=k_{M} \frac{\partial^{2} T_{M}}{\partial y^{2}}-2 \frac{\varepsilon_{M} \sigma}{h_{M}}\left(T_{M}^{4}-T_{0}^{4}\right) .
$$

Here $\varepsilon_{M}$ and $h_{M}$ are the emissivity and the thickness of the membrane, respectively. The factor of two in the radiation term arises because the membrane radiates from the top and bottom surfaces. If we let $\tau=T_{M}-T_{0}$, and approximate the radiation term with a linear 
Taylor expansion about $\tau=0$, then the radiation term becomes $\left(8 \varepsilon_{M} \sigma T_{0}^{3} / h_{M}\right) \tau$. By letting $\alpha=k_{M} /\left(\rho c_{P}\right)_{M}$, and $\beta=8 \varepsilon_{M} \sigma T_{0}^{3} / h_{M}\left(\rho c_{P}\right)_{M}$, Eq. (5) reduces to,

$$
\frac{\partial \tau}{\partial t}=\alpha \frac{\partial^{2} \tau}{\partial y^{2}}-\beta \tau
$$

with initial and boundary conditions,

$$
\tau[y, 0]=0, \quad \tau[0, t]=f[t], \quad \tau[\infty, t]=0 .
$$

Here the temperature in the membrane is assumed to be initially uniform. The temperature at the left boundary $(y=0)$ evolves as a function of time, while the right boundary $(y=\infty)$ remains fixed at the initial value. Comparing these conditions to the physical thermal cell, the initial condition is satisfied by letting the sensor equilibrate with its surroundings for an appropriate length of time (on the order of seconds for these sensors). The left boundary condition is given by the experimentally measured temperature history of the thermistor. The right boundary condition remains valid as long as the thermal diffusion length is smaller than the distance to the edge of the membrane. The linearization of the radiation term in the membrane is valid as long as the temperature difference between the membrane and its surroundings remains appropriately low.

To solve Eq. (6), we follow Sneddon's example for the solution to Eq. (6) without the radiation term [16]. Starting with an auxiliary problem, we first solve the case where the left boundary condition satisfies $\tau[0, t]=f\left[t^{\prime}\right]$, where $t^{\prime}$ is a fixed parameter, i.e., $f\left[t^{\prime}\right]$ is a constant. Let $\tau^{*}[y, t]$ be the solution to the auxiliary problem. Applying the Fourier sine transform,

$$
\theta[\psi, t]=\sqrt{\frac{2}{\pi}} \int_{0}^{\infty} \tau *[y, t] \sin [\psi y] d y,
$$

to Eq. (6) and taking into account the boundary conditions yields

$$
\frac{\partial \theta}{\partial t}+\left(\beta+\alpha \psi^{2}\right) \theta[\psi, t]=\sqrt{\frac{2}{\pi}} \alpha \psi f\left[t^{\prime}\right] .
$$

With $\theta[\psi, 0]=0$ from Eq. (6b), Eq. (8) can be solved with a result 


$$
\theta[\psi, t]=\sqrt{\frac{2}{\pi}} \frac{\alpha \psi f\left[t^{\prime}\right]}{\beta+\alpha \psi^{2}}\left(1-e^{-\left(\beta+\alpha \psi^{2}\right) t}\right) .
$$

Applying the inverse Fourier sine-transform yields the solution of the auxiliary problem

$$
\tau^{*}[y, t]=\frac{2}{\pi} \int_{0}^{\infty} \frac{\alpha \psi f\left[t^{\prime}\right]}{\beta+\alpha \psi^{2}}\left(1-e^{-\left(\beta+\alpha \psi^{2}\right) t}\right) \sin [\psi y] d \psi .
$$

Equation (10) represents the solution to Eq. (6a) for the case that $\tau^{*}[0, t]$ is constant. This result can be used with Duhamel's theorem to solve Eq. (6a) when the boundary condition is a function of time. According to Duhamel's theorem [16], the solution for the problem with the variable boundary condition, $\tau[0, t]=f[t]$, is related to the solution $\tau^{*}[y, t]$ for the fixed boundary condition $\tau[0, t]=f\left[t^{\prime}\right]$ by

$$
\tau[y, t]=\frac{\partial}{\partial t} \int_{0}^{t} \tau *\left[y, t-t^{\prime}\right] d t^{\prime} .
$$

Substituting Eq. (10) into Eq. (11) results in,

$$
\tau[y, t]=\frac{\partial}{\partial t} \int_{0}^{t} \frac{2}{\pi} \int_{0}^{\infty} \frac{\alpha \psi f\left[t^{\prime}\right]}{\beta+\alpha \psi^{2}}\left(1-e^{-\left(\beta+\alpha \psi^{2}\right)\left(t-t^{\prime}\right)}\right) \sin [\psi y] d \psi d t^{\prime} .
$$

Taking the derivative and simplifying gives,

$$
\tau[y, t]=\frac{2}{\pi} \alpha \int_{0}^{t} f\left[t^{\prime}\right] \int_{0}^{\infty} \psi \sin [\psi y] e^{-\left(\beta+\alpha \psi^{2}\right)\left(t-t^{\prime}\right)} d \psi d t^{\prime} .
$$

Using the result from reference [17],

$$
\int_{0}^{\infty} \psi \sin [\psi y] e^{-\alpha \psi^{2}\left(t-t^{\prime}\right)} d \psi=\frac{\sqrt{\pi}}{4} \frac{y e^{-\frac{y^{2}}{4 \alpha\left(t-t^{\prime}\right)}}}{\left(\alpha\left(t-t^{\prime}\right)\right)^{3 / 2}},
$$

and substituting into Eq. (13), yields,

$$
\tau[y, t]=\int_{0}^{t} f\left[t^{\prime}\right] \frac{y \operatorname{Exp}\left[\frac{-y^{2}}{4 \alpha\left(t-t^{\prime}\right)}-\beta\left(t-t^{\prime}\right)\right]}{2 \sqrt{\pi \alpha}\left(t-t^{\prime}\right)^{3 / 2}} d t^{\prime} .
$$

Equation (15) represents the temperature profile in the membrane for a given temperature history of the heating element. The first factor inside the integral is the forcing function, while the second factor is the Green's function of the problem. If the temperature history of the thermistor $f\left[t^{\prime}\right]$ is represented by an $n^{\text {th }}$ order polynomial with coefficients $\left\{a_{0}, a_{1}\right.$, 
$\left.a_{2}, \ldots, a_{n}\right\}$, then Eq. (15) can be expressed analytically, although the result becomes complicated quickly with increasing order of the polynomial. Taking the derivative of the resulting analytic function with respect to $y$ and evaluating at $y=0$, gives the temperature gradient in the membrane at the edge of the $\mathrm{CV}$ in terms of the polynomial coefficients $a_{i}$ :

$$
\left.\frac{\partial \tau}{\partial y}\right|_{y=0}=\sqrt{\frac{1}{\alpha}} \sum_{i=1}^{n} a_{i} b_{i}[t, \beta],
$$

where the $b_{i}[t, \beta]$ are functions of $t$ and $\beta$ alone. They follow from the operations described above and can be found in Appendix A for polynomials up to order five for $\beta>$ 0 and order seven for $\beta=0$. Since $\partial \tau / \partial y=\partial T_{M} / \partial y$, we substitute Eq. (16) into Eq. (4) and let $g[t]=\Sigma a_{i} b_{i}[t, \beta]$ to get,

$$
P=C_{P} r[t]+2 A_{M} \phi_{M} g[t]+2 A_{z} \varepsilon_{C V} \sigma\left(\left(f[t]+T_{0}\right)^{4}-T_{0}^{4}\right) .
$$

Here the effusivity is defined as $\phi=\sqrt{k \rho c_{P}}$. Equation (17) establishes the power balance of the $\mathrm{CV}$ in terms of the unknown thermal parameters $C_{P}, \phi_{M}$ and $\varepsilon_{C V}$. Data analysis will focus on using Eq. (17) to evaluate these thermal parameters.

\section{Finite Element Simulations}

A two-dimensional transient finite element model (FEM) has been created to simulate the heat flow in the thermal cell and to evaluate the accuracy of the analytical model. The model represents a cross-section of the sensor in the $y z$-plane (Fig. 2(b)), reduced by the mirror symmetry at the center of the heating element. The dimensions of the model are based on the actual thermal cell used in our experiments (Table 1). The left-end boundary condition, at the mirror plane, imposes zero heat flux, while the rightend boundary condition maintains the edge of the membrane at $T_{0}$. The top and bottom surfaces allow radiative heat loss to a blackbody at $T_{0}$. The initial condition is $T[0, y]=T_{0}$ everywhere.

In order to simulate a measurement, two finite element models are required: a model of a bare cell consisting of a silicon nitride membrane and a tungsten heater, and a 
model of a cell with a representative sample, in this case a $50 \mathrm{~nm}$ copper film. Based on experimental results presented in a subsequent section, the initial resistance of the heating element is set to $R_{0}=45 \Omega$, while the temperature coefficient of resistance is $\lambda=$ $1.65 \times 10^{-3} \mathrm{~K}^{-1}$. The remaining materials properties are based on literature values, shown in Table 2.

The finite element simulations have been performed with the commercial code ABAQUS. The heat transfer is modeled using the diffusive heat transfer element DC2D8, an 8-node biquadratic element. The $y$-spacing of the elements inside the $\mathrm{CV}$ is approximately $4 \mathrm{~nm}$, while the spacing in the membrane is approximately $21 \mathrm{~nm}$. The heater is represented by 4 elements through the film thickness; the membrane (membrane plus sample when present) is represented by 2 elements. Heat generation is accomplished via a user subroutine associated with the elements of the thermistor. A constant current $I$ ( $5 \mathrm{~mA}$ for the reference case and $10 \mathrm{~mA}$ for the sample case) and a resistance $R=R_{0}(1+$ $\left.\lambda\left(T-T_{0}\right)\right)$ determine the electrical power dissipated in the heating element. A time step of $0.05 \mathrm{~ms}$ is used in the simulations.

The output of the simulations is in the form of the nodal temperature history. The nodal temperatures show that the temperature variations through the thickness of the membrane and heating element are small $\left(\Delta T<0.1^{\circ} \mathrm{C}\right)$. The FEM neglects interface resistances between the layers in the $\mathrm{CV}$ as does the analytical model. This assumption can be justified by considering the worst case conditions of a heat flux through the interface equal to the entire power dissipated in the heater $(4.5 \mathrm{~mW})$ and a very low thermal conductance $\left(20 \mathrm{MW} / \mathrm{m}^{2} \mathrm{~K}\right)$ [1], which produces a temperature difference across the interface of just $0.001^{\circ} \mathrm{C}$. This temperature error is insignificant and can be neglected. Averaging the temperature of the nodes through the thickness of the model produces a temperature distribution along the $y$-direction. Figure 4 shows temperature distributions in a reference cell and in a sample cell with $50 \mathrm{~nm}$ of copper for a representative set of experimental conditions. These temperature distributions confirm the earlier assumption that the lateral temperature variation within the $\mathrm{CV}$ is small $\left(\Delta T<0.1^{\circ} \mathrm{C}\right)$. From these observations, we conclude that the temperature boundary condition $f[t]$ in the analytical model can be represented by the average temperature of 
the thermistor. This is equivalent to the actual experiments where the average temperature is measured. The temperature-time data generated by the finite element models (Fig. 5) are then analyzed using the method described in the next section to determine the heat capacity of the control volume $C_{P}$ and the effusivity of the membrane $\phi_{M}$.

\section{Data Reduction and Error Analysis}

A data reduction method based on the analytical model has been developed to analyze the FEM results and the experimental data. Analysis of the FEM data allows for verification of the data reduction method by accurate reproduction of input values; analysis of the measured data produces the desired materials properties that are the goal of this investigation. The following discussion is based on the FEM data, but the analysis of experimental data proceeds along the same line.

The data reduction begins with the temperature history of the thermistor obtained from the FEM calculations. To simulate experimental noise in the FEM results, a normally distributed temperature error $\sigma_{T}[p]$ was added to the FEM temperature results at each time step in the temperature report. The width of the $\sigma_{T}[p]$ distribution was representative of the measurement noise observed experimentally. Equation (17) can be used with this data set to estimate the thermal parameters $C_{P}$ and $\phi_{M}$. For each time step $t[N]$, the function $f\left[t^{\prime}\right]$ and the $a_{i}$ in Eq. (16) are found from a polynomial fit to the temperature history up to that particular point $N$. The fit is obtained by minimizing the merit function,

$$
\chi_{a}^{2}=\sum_{p=1}^{N}\left(\frac{T[p]-\left(a_{0}[N]+a_{1}[N] t[p]+\ldots+a_{n}[N] t^{n}[p]\right)}{\sigma_{T}[p]}\right)^{2} .
$$

The order of the polynomial is determined by the linearity of the temperature history. For the bare cell, $n=5$ is sufficient to represent the simulation results; for the cell with sample, $n=7$ is required. The merit function $\chi_{a}^{2}$ is minimized by applying the normal equations in a matrix formulation. Following this approach, we define the matrices, 


$$
v[N]=\left[\begin{array}{llll}
\sum_{p=1}^{N} \frac{1}{\sigma_{T}^{2}[p]} & \sum_{p=1}^{N} \frac{t[p]}{\sigma_{T}^{2}[p]} & \cdots & \sum_{p=1}^{N} \frac{t^{n}[p]}{\sigma_{T}^{2}[p]} \\
\sum_{p=1}^{N} \frac{t[p]}{\sigma_{T}^{2}[p]} & \sum_{p=1}^{N} \frac{t^{2}[p]}{\sigma_{T}^{2}[p]} & & \\
\vdots & & \ddots & \\
\sum_{p=1}^{N} \frac{t^{n}[p]}{\sigma_{T}^{2}[p]} & & & \sum_{p=1}^{N} \frac{t^{2 n}[p]}{\sigma_{T}^{2}[p]^{2}}
\end{array}\right],
$$

and

$$
\omega[N]=\left[\sum_{p=1}^{N} \frac{T[p]}{\sigma_{T}^{2}[p]} \quad \sum_{i=1}^{N} \frac{T[p] t[p]}{\sigma_{T}^{2}[p]} \quad \ldots \quad \sum_{i=1}^{N} \frac{T[p] t^{n}[p]}{\sigma_{T}^{2}[p]}\right]
$$

Then the $a_{i}$ are given by,

$$
a_{i}[N]=\sum_{j=1}^{n+1} v_{(i+1) j}^{-1}[N] \omega_{j}[N]
$$

Equation (21) is used to evaluate the function $g[t]$ in Eq. (17). The error $\sigma_{g}$ on $g[t]$ is then given by

$$
\sigma_{g}^{2}=\sum_{j=1}^{n} \sum_{i=1}^{n} \frac{\partial g}{\partial a_{i}} \frac{\partial g}{\partial a_{j}} v_{(i+1)(j+1)}^{-1}
$$

The covariances of the $a_{i}$ parameters are significant and must be included for an accurate estimation of $\sigma_{g}$.

The average heating rate of the control volume, $r[t]$, is the derivative of the temperature history. In the data analysis, this derivative is calculated by performing a linear least square fit of the temperature data around the time step of interest. The fit is found by minimizing the merit function

$$
\chi_{c}^{2}[N]=\sum_{p=N-M}^{N+M}\left(\frac{T[p]-\left(c_{0}[N]+c_{1}[N] t[p]\right)}{\sigma_{T}[p]}\right)^{2},
$$

using the normal equations in a matrix formulation. The corresponding right hand side (RHS) of Eq. (19) is now a 2x2 matrix, and the RHS of Eq. (20) is a 2-component vector. 
In both equations, the upper and lower limits for the summations are the same as in Eq. (23), i.e., $N-M$ and $N+M$, respectively. The heating rate is then,

$$
r[N]=c_{1}[N]=v_{21}^{-1}[N] \omega_{1}[N]+v_{22}^{-1}[N] \omega_{2}[N]
$$

with associated error,

$$
\sigma_{r}^{2}[N]=v_{22}^{-1}[N]
$$

Before $C_{P}$ and $\phi_{M}$ can be determined from Eq. (17), it is necessary to know $\beta$ and $\varepsilon_{C V}$. We will show later in this section how these parameters can be estimated from experiments. In many cases, however, the effects of $\beta$ and $\varepsilon_{C V}$ are small enough that they can be neglected without significant loss of accuracy. Alternately literature values can be used. Figure 6 shows the radiation loss from the $\mathrm{CV}$ and the membrane of a bare thermal cell relative to the total power input for a heating rate of $7.5 \mathrm{~K} / \mathrm{ms}$. In the analysis of the FEM data, the FEM input values were used for $\varepsilon_{C V}$ and $\beta$. Because radiation from the membrane in the sample cell was insignificant, $\beta$ was taken as zero for the sample cell.

All parameters in Eq. (17) are now known except for $C_{P}$ and $\phi_{M}$, which are determined by a least squares fit of Eq. (17) to the $P$ data. The error on the electrical power, $\sigma_{P}$, is typically much smaller than the errors on $g[t]$ and $r[t]$. To account for the uncertainty in these two terms, an effective error is defined for the electrical power, $\sigma_{e f f}$, as [18],

$$
\sigma_{\text {eff }}^{2}=\sigma_{P}^{2}+\left(\frac{\partial P}{\partial r} \sigma_{r}\right)^{2}+\left(\frac{\partial P}{\partial g} \sigma_{g}\right)^{2}
$$

and used in the merit function for the fit. The partial derivatives in Eq. (26) require some knowledge about the unknown parameters $C_{P}$ and $\phi_{M}$. For the FEM data, the input values are used; for the experimental analysis, $C_{P}$ and $\phi_{M}$ are first estimated with a least squares fit using $\sigma_{P}$ only and then $\sigma_{\text {eff }}$ is evaluated using the estimated values. The merit function for the least squares fit of the thermal parameters is defined as, 


$$
\chi_{t}^{2}=\sum_{p=1}^{N}\left(\frac{P[p]-2 A_{z} \varepsilon_{C V} \sigma\left(\left(f[p]+T_{0}\right)^{4}-T_{0}^{4}\right)-\left(C_{P}[N] r[p]+2 A_{M} \phi_{M}[N] g[p]\right)}{\sigma_{e f f}[p]}\right)^{2} .
$$

Equation (27) is minimized as described above to produce the best-fit values of $C_{P}$ and $\phi_{M}$. These terms can be written explicitly as,

$C_{P}[N]=\frac{1}{\Gamma[N]}\left(\sum_{p=1}^{N} \frac{P_{e f f}[p] r[p]}{\sigma_{e f f}^{2}[p]} \sum_{p=1}^{N} \frac{g^{2}[p]}{\sigma_{e f f}^{2}[p]}-\sum_{p=1}^{N} \frac{g[p] r[p]}{\sigma_{e f f}^{2}[p]} \sum_{p=1}^{N} \frac{P_{e f f}[p] g[p]}{\sigma_{e f f}^{2}[p]}\right)$,

and

$$
\phi[N]=\frac{1}{2 A_{M} \Gamma[N]}\left(\sum_{p=1}^{N} \frac{r^{2}[p]}{\sigma_{e f f}^{2}[p]} \sum_{p=1}^{N} \frac{P_{e f f}[p] g[p]}{\sigma_{e f f}^{2}[p]}-\sum_{p=1}^{N} \frac{P_{e f f}[p] r[p]}{\sigma_{e f f}^{2}[p]} \sum_{p=1}^{N} \frac{r[p] g[p]}{\sigma_{e f f}^{2}[p]}\right),
$$

with associated errors,

$$
\sigma_{C_{P}}^{2}[N]=\frac{1}{\Gamma[N]} \sum_{p=1}^{N} \frac{g^{2}[p]}{\sigma_{e f f}^{2}[p]},
$$

and

$$
\sigma_{\phi}^{2}[N]=\left(\frac{1}{2 A_{M}}\right)^{2} \frac{1}{\Gamma[N]} \sum_{p=1}^{N} \frac{r^{2}[p]}{\sigma_{e f f}^{2}[p]} .
$$

In these equations,

$$
\Gamma[N]=\sum_{p=1}^{N} \frac{r^{2}[p]}{\sigma_{e f f}^{2}[p]} \sum_{p=1}^{N} \frac{g^{2}[p]}{\sigma_{e f f}^{2}[p]}-\left(\sum_{p=1}^{N} \frac{r[p] g[p]}{\sigma_{e f f}^{2}[p]}\right)^{2},
$$

and $P_{\text {eff }}=P-Q_{z}$. Analysis results of $C_{P}$ and $\phi_{M}$ are plotted along with input values for the bare cell in Figs. 7(a) and 7(b), while the values for the cell with the sample are plotted in Figs. 7(c) and 7(d), respectively. It is evident that the analysis results converge rapidly to the input values. The average analysis result is calculated with an error-weighted mean and shown in Table 3 along with input values and reproducibility error. A reproducibility error of approximately $3 \%$ or less provides confidence in the analytical model and the data reduction scheme. The error analysis presented here is extended to a sensitivity analysis in Appendix B that provides relative errors for the effusivity, heat capacity, and 
thermal conductivity. These relative errors can be used to judge the capability of the device and to guide experimental work on other materials systems.

The FEM results have also been used to simulate measurement of the emissivity $\varepsilon_{C V}$ of the $\mathrm{CV}$. This is achieved by heating narrow $(400 \mu \mathrm{m})$ and wide $(1000 \mu \mathrm{m})$ heating elements at approximately the same rate of $20 \mathrm{~K} / \mathrm{ms}$ to a temperature of $400^{\circ} \mathrm{C}$. Under these conditions the difference in conductive loss between the two sensors is small, while the difference in radiation loss is significant. By taking the difference in the power balance for each heater (Eq. 17) and assuming that conductive losses cancel exactly, the effective emissivity of the CV can be written as,

$$
\varepsilon_{C V}=\frac{\Delta P-\Delta\left(C_{P}(d T / d t)\right)}{l \Delta w \sigma\left(T^{4}-T_{0}^{4}\right)} .
$$

Using this method, the effective emissivity of a bare cell is determined to be $\varepsilon_{C V}=0.104$, which differs from the input value by $1 \%$ (Fig. 8). This method of evaluation can be used when the $\varepsilon_{C V}$ term is significant and measured values are preferred over literature values. The emissivity of the tungsten film, which is used for the heating element, can be obtained from this method by depositing tungsten as a sample film. In this case the $\varepsilon_{C V}$ is the emissivity of tungsten. Once the emissivity of tungsten is known, then the emissivity of the membrane can be estimated from the effective emissivity of the bare cell CV.

Determination of $C_{P}$ by fitting Eq. (17) to the power data requires a value for $\varepsilon_{C V}$. Determination of $\varepsilon_{C V}$ from Eq. (33), on the other hand, requires a value for $C_{P}$. For the finite element model the input values can be used, but for experiments these values are not known a priori. They are, however, easily determined iteratively. More specifically, Eq. (17) is fitted to the experimental data using literature values for $\varepsilon_{C V}$; then the $C_{P}$ result from this fit is used to calculate an experimental value for $\varepsilon_{C V}$. This value of $\varepsilon_{C V}$ is then used in Eq. (17) to produce the final result. Because the effect of radiation is very small in the temperature and heating rate range considered, one iteration is sufficient. 
The accurate reproduction of FEM input values with the analytical model and data reduction scheme validates the assumptions made in the analytical model and lends credibility to the measurement approach. The same analysis method can now be applied to experimental results obtained for real devices. The following sections describe how the device is made and the measurements are performed, as well as the results of the measurements.

\section{Device Fabrication}

The fabrication process starts with (100) oriented Si wafers, $200 \mathrm{~mm}$ in diameter and polished on one side. These wafers are delivered with a coating of approximately $80 \mathrm{~nm}$ of $\mathrm{Si}_{3} \mathrm{~N}_{4}$ grown on both sides using low-pressure chemical vapor deposition (Fig. 9(a)). Special care is taken throughout the fabrication process to protect the $\mathrm{Si}_{3} \mathrm{~N}_{4}$ on the polished side of the wafer. This film will eventually form the membranes of the nTTA and even shallow scratches result in ruptured membranes.

Each Si wafer is cleaved into seven $55 \mathrm{~mm} \times 55 \mathrm{~mm}$ square substrates. The substrates are rinsed in deionized water and blown with nitrogen to remove any particles. Then $125 \mathrm{~nm}$ of tungsten and $1.2 \mu \mathrm{m}$ of copper are deposited on the polished side of a square substrate using direct current (DC) magnetron sputtering (Fig. 9(b)). Immediately prior to film deposition, the substrates are sputter-cleaned using an Ar plasma to remove any contamination and to improve adhesion of the sputtered coatings.

With the materials necessary for forming the sensors in place, material is now selectively removed to form the appropriate structures using standard photolithography and etch processes. After the wafer is baked at $150^{\circ} \mathrm{C}$ for $5 \mathrm{~min}$, Shipley 1805 photoresist (S1805) is spin-coated and patterned on both sides of the wafer. The front side of the substrate is exposed to UV light through a mask with the metallization artwork, and the backside is exposed through a mask with the cavity window artwork. Both sides of the substrate are developed simultaneously in Microposit CD-30 for 1 minute (Fig. 9(c)). The 
$\mathrm{Si}_{3} \mathrm{~N}_{4}$ on the backside of the wafer is reactively etched in $\mathrm{CF}_{4}$ to create rectangular openings in the silicon nitride layer. Copper is etched in a solution of phosphoric, nitric, and acetic acid at $50^{\circ} \mathrm{C}$. The $\mathrm{Cu}$ etch exposes the underlying $\mathrm{W}$, which is then etched in $30 \% \mathrm{H}_{2} \mathrm{O}_{2}$ at $50^{\circ} \mathrm{C}$ (Fig. $9(\mathrm{~d})$ ). Both etch steps take approximately three minutes. After the wet-etch processes, the remaining resist is exposed and removed. Next S1805 is reapplied to the metallization side and patterned with the rectangular cavity artwork (Fig. 9(e)). Copper is then etched from the membrane area, leaving only $\mathrm{W}$ within the area that will form the membrane. After patterning of the metallization, the freestanding membranes are created by anisotropically etching the $\mathrm{Si}$ in a solution of $15 \mathrm{~g} \mathrm{KOH}$ in 50 $\mathrm{ml} \mathrm{H}_{2} \mathrm{O}$ at $85^{\circ} \mathrm{C}$ for approximately 9 hours. The patterned $\mathrm{Si}_{3} \mathrm{~N}_{4}$ coating on the backside of the substrate serves as a hard mask for this step. During this procedure, the metallization is protected by a sample holder that exposes the backside of the device to the $\mathrm{KOH}$ solution, while isolating the front side from the $\mathrm{KOH}$ solution. For added protection, a layer of Cyclotene resist (Dow Chemical) is spin-coated on top of the metallization and cured at $130^{\circ} \mathrm{C}$ for $70 \mathrm{~min}$. After the $\mathrm{Si}$ etch, the Cyclotene is removed in a bath of Primary Stripper A at $75^{\circ} \mathrm{C}$ for 1 hour (Fig. 9(f)). The device fabrication process is completed with an anneal at $450{ }^{\circ} \mathrm{C}$ for 8 hours in a vacuum furnace with a base pressure of $10^{-5} \mathrm{~Pa}$.

\section{Measurement Setup and Calibration of Thermistor}

nTTA measurements are controlled and recorded with a personal computer and a National Instruments PCI-6221 data acquisition card (DAQ) (Fig. 10). The DAQ is used to send a control voltage to a voltage-to-current converter, with a linear mapping of $1 \mathrm{~V}$ to $10 \mathrm{~mA}$. The current source consists of a precision operational amplifier (OPA227), a power operational amplifier (OPA549), and a differential amplifier (INA133) arranged in a modified Howland configuration. It is powered by a Protek 3030D dual DC power supply running in series mode, providing a constant $30 \mathrm{~V}$ controlled power. Excluding internal losses, the current supply is limited by approximately $20 \mathrm{~V}$ of compliance and can supply $100 \mathrm{~mA}$ of current. 
The output of the current source is monitored by the DAQ via the voltage drop $V_{I}$ across a $100 \Omega$ precision resistor $R_{I}$. The DAQ also reads the voltage drop $V$ across the thermistor. This is shown in schematic form in Fig. 10 and a typical result is shown in Fig. 11. All signals are recorded at a sampling rate of $100 \mathrm{kHz}$ and with a 16-bit resolution. Voltage signals are used to determine the resistance of the thermistor by $R=$ $R_{I} V / V_{I}$ and the electrical power dissipated in the thermistor by $P=V_{I} V / R_{I}$. The temperature of the thermistor is then calculated from,

$$
T=T_{0}+\frac{\Delta R}{\lambda R_{0}}
$$

where $\lambda$ is the temperature coefficient of resistance of the heating element, $R_{0}$ is the room temperature resistance, $\Delta R$ is the change in resistance, and $T_{0}$ is the ambient temperature.

Prior to depositing sample material onto the nTTA substrate, the thermal sensors need to be calibrated. To measure the temperature coefficient of resistance, the nTTA substrate is placed in an oven and stepped through a temperature range, while the temperature of the substrate is measured with a thermocouple. The resistance of the thermistor is recorded at each temperature step by applying a $1 \mathrm{~mA}$ monitoring current for $20 \mathrm{~ms}$. This current pulse causes a small amount of Joule heating $\left(\sim 0.3{ }^{\circ} \mathrm{C}\right)$. To eliminate this effect, $R$ is measured as a function of time and back extrapolated with a linear fit. $R_{0}$ and the ratio $\Delta R / \Delta T$ are determined from a linear least squares fit of the resistance data as a function of temperature. The value of $\lambda$ is then calculated using Eq. (34), yielding a value of $(1.65 \pm 0.02) \times 10^{-3} \mathrm{~K}^{-1}$ for the $\mathrm{W}$ thermistors in the cells. The value of $\lambda$ is typically determined for one cell on each substrate, while the value of $R_{0}$, is measured for each cell on a substrate.

All thermal transport measurements are conducted in a vacuum chamber with a vacuum level of $10^{-3} \mathrm{~Pa}$ to eliminate convection losses. Bare cells with narrow heating elements are subjected to a current of $5 \mathrm{~mA}$, while the wide heating elements receive an $80 \mathrm{~mA}$ pulse. The voltage response of the thermistor is recorded and transformed into a temperature history. This temperature history is then analyzed as described previously. 
Emissivity measurements are performed in a similar fashion. In this case, however, the current of the narrow thermistor is varied (11-12 $\mathrm{mA})$ to match the temperature history of the neighboring wide thermistor. After bare cell measurements are completed, samples are deposited on the thermal cells and the measurements are repeated.

\section{Sample Fabrication and Measurements}

We have demonstrated the capability of the nTTA device by measuring the thermal transport properties of thin $\mathrm{Cu}$ films as a function of film thickness. After the sensors in an nTTA device were characterized as described in the previous section, $\mathrm{Cu}$ films were deposited using DC magnetron sputtering and a $50.8 \mathrm{~mm}$ diameter $\mathrm{Cu}$ target. The depositions were performed at a power of $100 \mathrm{~W}$ and a pressure of $0.67 \mathrm{~Pa}$ using $\mathrm{Ar}$ as a working gas. The deposition rate was calibrated at each sensor location by depositing for a known length of time and measuring the resulting thickness with a Veeco Dektak profilometer. The thickness of each sample was then determined from the sample deposition time. The substrate was not rotated during the deposition so that the natural sputtering flux distribution would create a $\mathrm{Cu}$ thickness gradient along the columns of the nTTA (Fig. 1). A larger thickness gradient was created between the columns of the nTTA by stepping a shadow mask from left to right (Fig. 1). This process required a vacuum break and proceeded in such a way that the leftmost column (cells 1-5) had four discrete layers of copper, the next (cells 6-10) had three, the middle (cells 11-15) had two, and the two rightmost columns (cells 16-25) had just one layer. Cells 16-20 have narrow thermistors and cells 21-25 have wide thermistors. Neighboring thermistors in these columns form emissivity measurement pairs. Emissivity measurement pairs had the same $\mathrm{Cu}$ film thickness to ensure that the conductive heat losses would cancel as required for forming Eq. (33). The copper film thicknesses that resulted from this process are shown in Figure 12.

As with the bare thermal cells, the thermal transport measurements on the $\mathrm{Cu}$ coated sensors were performed in vacuum. The currents that were used for the measurements were chosen to approximately match the heating rates of the bare thermal 
cell measurements and can be found in Table 4. The emissivity measurements were performed by varying the current applied to the narrow thermistor of the pair to match the heating rate of the wide thermistor.

\section{Results and Discussion}

Initial resistance measurements taken at various instances throughout the experimentation demonstrate that the tungsten thermistors are stable during storage and low-temperature measurements, up to $450^{\circ} \mathrm{C}$, the temperature at which the device was annealed. The error on each $R_{0}$ measurement is approximately $8 \times 10^{-3} \Omega$, while the standard deviation of the $R_{0}$ results for cells $1-20$ is $2 \Omega$ (i.e. $4 \%$ ). Since the measurement error is much smaller than the standard deviation of the group, we conclude that the cellto-cell variation in resistance is real and most likely caused by slight variations in the thermistor dimensions as a result of the fabrication process.

With $R_{0}$ known for each cell, the temperature history of the CVs can be calculated from the voltage measurements using Eq. (34). Representative temperature curves for a bare cell and a cell with sample are shown in Fig. 13(a). Typical heating rates are shown in Fig. 13(b). The effective emissivity of the CV was determined from the heating curves of the narrow-wide thermistor pairs 16-21, 17-22, and 20-25. Pairs 18-23 and 19-24 were not included because cell 23 was broken and the data obtained from cell 24 was corrupted. The measured emissivity values are $\varepsilon_{C V}=0.14 \pm 0.01$ for the bare cells and $\varepsilon_{C V}$ $=0.17 \pm 0.01$ for the cells with $\mathrm{Cu}$ samples. These results compare well with literature values of $0.10-0.17$ and $0.03-0.15$ respectively [19-22]. The literature results vary considerably, typically explained by surface conditions. Our $\mathrm{Cu}$ films emit around the upper end of reported emissivity values, which is most likely due to slight oxidation of the film surface. The temperature curves of the other sensors were analyzed to determine $C_{P}$ and $\phi_{M}$ using the appropriate emissivity values listed above. Figure 14 shows representative fits of Eq. (17) to the power dissipated in the thermistors by minimizing the merit parameter $\chi_{t}$ (Eq. 27). Typical $C_{P}$ results for bare cell and cell with $61 \mathrm{~nm} \mathrm{Cu}$ sample are shown in Figs. 15(a) and (b) respectively. Typical $\phi_{M}$ results for the same cell 
conditions are shown in Fig. 15(c). It is evident that after some initial variability the parameters quickly converge to specific values. It should be noted that the $C_{P}$ result for the cell with the $\mathrm{Cu}$ sample was obtained by doubling the current over the $\phi_{M}$ measurements (Table 4) and reducing the considered time scale (1 ms) because of the large amount of heat conducted away by the $\mathrm{Cu}$ films.

The results for all bare cells and cells with samples are compiled in Figure 16. The $C_{P}$ results of the bare cells (Fig. 16(a)) show a similar distribution as the $R_{0}$ results with a standard deviation of approximately $3.7 \%$. This observation suggests that the distribution is again caused by variability in the fabrication process and not measurement error. The average value of the heat capacity, $126 \pm 6 \mathrm{~nJ} / \mathrm{K}$, compares well to the value of $123 \mathrm{~nJ} / \mathrm{K}$ obtained from the literature values of the constituents (See Table 2). The average measured effusivity value of the bare membrane is $2580 \pm 90 \mathrm{~J} / \mathrm{m}^{2} \mathrm{~s}^{1 / 2} \mathrm{~K}$, which compares closely with reported silicon nitride values on the order of $2600 \mathrm{~J} / \mathrm{m}^{2} \mathrm{~s}^{1 / 2} \mathrm{~K}$ [23]. The results for the cell with the $\mathrm{Cu}$ samples (Fig. 16) show the expected trend considering the $\mathrm{Cu}$ film thickness. The total heat capacity $C_{P}$ is an extrinsic materials property and the variation shown is caused by a change in sample mass; $\phi_{M}$ is an intrinsic materials property and the variation is caused by a change of the membrane effusivity as the film thickness varies.

The experimental values of $C_{P}$ and $\phi_{M}$ can be used to determine the volumetric heat capacity and the thermal conductivity of the $\mathrm{Cu}$ films: The volumetric heat capacity $\left(\rho c_{P}\right)$ of the films is readily found by taking the difference between the total heat capacities of the cells with and without samples and by normalizing that value with the $\mathrm{Cu}$ sample volume. The average experimental value thus obtained is $(3.7 \pm 0.2) \times 10^{6}$ $\mathrm{J} / \mathrm{m}^{3} \mathrm{~K}$, which is larger than the reported bulk value of $2.6 \times 10^{6} \mathrm{~J} / \mathrm{m}^{3} \mathrm{~K}$ [19]. Similar increases in specific heat for nanocrystalline copper have been reported previously [24, 25]. The error in the volumetric heat capacity of each $\mathrm{Cu}$ sample is relatively large for the thinnest samples, approximately $25 \%$, and reduces with film thickness to approximately $10 \%$ for the thickest samples. Errors result from the small volume of material measured and the significant amount of heat conducted away through the $\mathrm{Cu}$ film - in a sense $\mathrm{Cu}$ is 
probably one of the most challenging materials for these experiments (e.g., Appendix B). Further increasing the heating rates and decreasing the measurement times reduces this error. Samples with smaller thermal conductivity evidently result in smaller $\left(\rho c_{P}\right)$ errors. If necessary, very accurate and precise measurements of the heat capacity can also be made with a parallel nano-scanning calorimeter [26], a device similar to the nTTA but with slightly different thermistor and sample geometry.

The thermal conductivity of the copper films is calculated as,

$$
k_{C u}=\frac{\left(h_{S} \phi_{S}\right)^{2}-h_{S i N} k_{S i N}\left(h \rho c_{P}\right)_{C u}-\left(h_{S i N} \phi_{S i N}\right)^{2}}{\left.h_{C u}\left(h \rho c_{P}\right)_{C u}+\left(h \rho c_{P}\right)_{S i N}\right)},
$$

where the subscript $S$ refers to a value for a $\mathrm{Si}_{3} \mathrm{~N}_{4} / \mathrm{Cu}$ multilayer membrane (Fig. 17). Also shown in Fig. 17 are additional measurements for thicker multilayer $\mathrm{Cu}$ films (approximately 180 to $480 \mathrm{~nm}$ ) obtained from a second nTTA device. Evidently the error introduced by any uncertainty in the copper heat capacity is quite small for the thinnest films and it increases slightly with increasing film thickness (Eq. 35). The thermal conductivity of the copper films increases non-monotonically from approximately 15 to $300 \mathrm{~nm}$, where the values apparently plateau around $300 \mathrm{~W} / \mathrm{m} \mathrm{K}$, well below the bulk value of $410 \mathrm{~W} / \mathrm{m} \mathrm{K}$ [19]. This behavior can be explained by the multilayer structure of the $\mathrm{Cu}$ films and by scattering of electrons from the surfaces and interfaces of the copper films (Fig. 18).

To gain further insight into these results we consider the model by Qiu and Tien [27],

$$
\frac{k_{\text {film }}}{k_{\text {bulk }}}=\left(1+\frac{3}{8} \frac{B}{\eta}+\frac{7}{5}\left(\frac{G}{1-G}\right) \frac{B}{D}\right)^{-1} .
$$

Equation (36) relates the thermal conductivity of a metal film to its bulk thermal conductivity based on the bulk electron mean free path $B$, the film thickness $\eta$, the grain boundary reflection coefficient $G$, and the grain boundary diameter $D$. This formulation of the model assumes diffuse reflections at the interfaces. We use literature values for $B$ 
and $G-B=42 \mathrm{~nm}[28], G=0.25$ [27] - and take $D=\eta$ based on our TEM observations. Finally we define the normalized conductivity as $\kappa=k_{f i l m} / k_{b u l k}$, and the effective normalized thermal conductivity of a multilayer film as,

$$
\kappa_{e f f}=\frac{\eta_{1} \kappa_{1}+\eta_{2} \kappa_{2}+\ldots+\eta_{n} \kappa_{n}}{\eta_{1}+\eta_{2}+\ldots+\eta_{n}}
$$

for an $n$-layer stack of films, where $\kappa_{i}$ and $\eta_{i}$ represent the thermal conductivity and thickness of the $i^{\text {th }}$ layer. Our measured results and the model predictions are compared in Fig. 19. The good agreement indicates that the internal copper interfaces and grain boundaries indeed act as scattering surfaces for electrons. They clearly replicate the nonmonotonic behavior and the relatively low plateau value of the films, indicating that both are caused by the multilayered structure of the $\mathrm{Cu}$ coatings.

\section{Conclusions}

Many capable in-plane thermal conductivity measurement devices exist. Some of these devices have better accuracy than the nTTA because they use multiple measurement points. Other devices that rely on a steady state have no dependence on volumetric heat capacity, simplifying data reduction. The nTTA technique, however, has the unique feature that it can be used as a combinatorial device. To accomplish this, it is necessary to trade multiple measurement locations for a single line measurement. Extraction of material parameters can be accomplished using an analytical model for the thermal behavior of the sensors. This analytical model was verified with finite element simulations and input parameters were reproduced with high accuracy. The capabilities of the nTTA were demonstrated by measuring thermal transport properties of $\mathrm{Cu}$ films with thicknesses less than the mean free path of the dominant heat carriers.

\section{Acknowledgments}

The authors would like to thank Jim MacArthur for designing the current source, Jae Hyun Kim for helping develop the finite element model, Ohad Rosenberg for his supplemental measurements of the three thickest copper films, Yizhong Huang for his 
TEM work, and Yves Chretien for discussions on error propagation. The work presented in this paper was supported by the Air Force Office of Scientific Research (AFOSR) under Grant FA9550-08-1-0374, and by the Materials Research Science and Engineering Center (MRSEC) at Harvard University. It was performed in part at the Center for Nanoscale Systems (CNS), a member of the National Nanotechnology Infrastructure Network (NNIN), which is supported by the National Science Foundation under NSF Award No. ECS-0335765. CNS is part of the Faculty of Arts and Sciences at Harvard University. 


\section{Appendix A: Coefficients of Equation (16)}

Expressions for the coefficients in Eq. (16) for $\beta>0$ and $n=5$.

$$
\begin{aligned}
& b_{1}=t^{1 / 2} \frac{e^{-\beta t}}{\sqrt{\pi}}+\left(\frac{1}{2} \beta^{-1 / 2}+\beta^{1 / 2} t\right) \operatorname{Erf}[\beta t] \\
& b_{2}=\left(\frac{1}{2} \beta^{-1} t^{1 / 2}+t^{3 / 2}\right) \frac{e^{-\beta t}}{\sqrt{\pi}}+\left(-\frac{1}{4} \beta^{-3 / 2}+\beta^{-1 / 2} t+\beta^{1 / 2} t^{2}\right) \operatorname{Erf}[\sqrt{\beta t}] \\
& b_{3}=\left(-\frac{3}{4} \beta^{-2} t^{1 / 2}+\beta^{-1} t^{3 / 2}+t^{5 / 2}\right) \frac{e^{-\beta} t}{\sqrt{\pi}}+\left(\frac{3}{8} \beta^{-5 / 2}-\frac{3}{4} \beta^{-3 / 2} t+\frac{3}{2} \beta^{-1 / 2} t^{2}+\beta^{1 / 2} t^{3}\right) \operatorname{Erf}[\sqrt{\beta t}] \\
& b_{4}=\left(\frac{15}{8} \beta^{-3} t^{1 / 2}-\frac{7}{4} \beta^{-2} t^{3 / 2}+\frac{3}{2} \beta^{-1} t^{5 / 2}+t^{7 / 2}\right) \frac{e^{-\beta t}}{\sqrt{\pi}}+\left(-\frac{15}{16} \beta^{-7 / 2}+\frac{3}{2} \beta^{-5 / 2} t-\frac{3}{2} \beta^{-3 / 2} t^{2}+2 \beta^{-1 / 2} t^{3}+\beta^{1 / 2} t^{4}\right) \operatorname{Erf}[\sqrt{\beta} t] \\
& b_{5}=\left(-\frac{105}{16} \beta^{-4} t^{1 / 2}+5 \beta^{-3} t^{3 / 2}-3 \beta^{-2} t^{5 / 2}+2 \beta^{-1} t^{7 / 2}+t^{9 / 2}\right) \frac{e^{-\beta t}}{\sqrt{\pi}}+\left(\frac{105}{32} \beta^{-9 / 2}-\frac{75}{16} \beta^{-7 / 2} t+\frac{15}{4} \beta^{-5 / 2} t^{2}-\frac{5}{2} \beta^{-3 / 2} t^{3}+\frac{5}{2} \beta^{-1 / 2} t^{4}+\beta^{1 / 2} t^{5}\right) \operatorname{Erf}[\sqrt{\beta t}]
\end{aligned}
$$

Expressions for the coefficients in Eq. (16) for $\beta=0$ and $n=7$.

$$
\begin{aligned}
& b_{1}=2 \frac{t^{1 / 2}}{\sqrt{\pi}} \\
& b_{2}=\frac{8}{3} \frac{t^{3 / 2}}{\sqrt{\pi}} \\
& b_{3}=\frac{16}{5} \frac{t^{5 / 2}}{\sqrt{\pi}} \\
& b_{4}=\frac{128}{35} \frac{t^{7 / 2}}{\sqrt{\pi}} \\
& b_{5}=\frac{256}{63} \frac{t^{9 / 2}}{\sqrt{\pi}} \\
& b_{6}=\frac{1024}{231} \frac{t^{11 / 2}}{\sqrt{\pi}} \\
& b_{7}=\frac{2048}{429} \frac{t^{13 / 2}}{\sqrt{\pi}}
\end{aligned}
$$




\section{Appendix B: Measurement Sensitivity Analysis}

Equations (28-32) from the main body of the paper can be used to define the relative errors for the heat capacity and effusivity, i.e. $\delta_{C_{P}}=\sigma_{C_{P}} / C_{P}$ and $\delta_{\phi}=\sigma_{\phi} / \phi$, which are metrics for the sensitivity of the technique. Figure B1 shows contour plots of the relative errors as a function of the thermal parameters assuming a constant heating rate of $3000 \mathrm{~K} / \mathrm{s}$ for $5 \mathrm{~ms}$ and a temperature error $\sigma_{T}=0.1{ }^{\circ} \mathrm{C}$. It is evident from the figure that the relative error on the effusivity increases with increasing $C_{P}$ and decreasing $A_{M} \times \phi_{M}$. Conversely, the relative error on the heat capacity decreases with increasing $C_{P}$ and decreasing $A_{M} \times \phi_{M}$. Similar plots are readily created for other experimental conditions to evaluate the effects on measurement sensitivity of experimental parameters such as the heating rate or sensor dimensions.

The relative errors on $C_{P}$ and $\phi_{M}$ can be used to estimate the errors on the thermal properties of the materials. Equation (B1) defines the error on the heat capacity of a $\mathrm{Cu}$ sample deposited on the nTTA,

$$
\delta_{C_{P}, C u}=\frac{\sqrt{\left(\delta_{C_{P}, S} C_{P, S}\right)^{2}+\left(\delta_{C_{P}, B} C_{P, B}\right)^{2}}}{C_{P, S}-C_{P, B}} .
$$

Here the subscripts B and $\mathrm{S}$ indicate a bare thermal cell and a cell with sample, respectively. Applying Eq. (B1) to the example of a $61 \mathrm{~nm}$ thick $\mathrm{Cu}$ sample gives, $C_{P, B}=$ $126 \mathrm{~nJ} / \mathrm{K}$ and $C_{P, S}=173 \mathrm{~nJ} / \mathrm{K}$, with respective errors from Fig. (B1) $\delta_{C_{P}, B}=1.6 \%$ and $\delta_{C_{P}, S}=2.7 \%$, so that $\delta_{C_{P}, C u}=11 \%$. The determination of $\left(\rho c_{P}\right)$ requires a volume measurement of the sample, which has a relative error of $10 \%$. Combining these errors gives a relative error of $15 \%$ on $\left(\rho c_{P}\right)$ for the $61 \mathrm{~nm} \mathrm{Cu}$ film. The relative errors on $(\rho$ $c_{P}$ ) for $23 \mathrm{~nm}$ and $120 \mathrm{~nm} \mathrm{Cu}$ films are estimated at $22 \%$ and $12 \%$ respectively.

Similarly, the relative error of the thermal conductivity of thin-films deposited on the nTTA can be defined in terms of the errors on the measured thermal properties. The relative error on the thermal conductivity of the sample film follows from the effective thermal conductivity of a multilayer film (Eq. 35). Using the example of the $61 \mathrm{~nm} \mathrm{Cu}$ film, from Fig. (B1) $\delta_{\phi, S}=0.75 \%, \delta_{\left(\rho c_{P}\right), C u}=15 \%, \delta_{\phi, S i N}=2.9 \%$, and $\delta_{\left(\rho c_{P}\right), S i N}=1.6 \%$, 
produces an error on the thermal conductivity of the $\mathrm{Cu}$ film of $\delta_{k, C u}=8.6 \%$, in good agreement with the experimental results. The error analysis can be used to estimate errors in thermal parameters and thermal materials properties; it serves as a guide when planning measurements on other materials systems and for optimizing the measurement technique. 


\section{References}

[1] D.G. Cahill, W.K. Ford, K.E. Goodson, G.D. Mahan, A. Majumdar, H.J. Maris, R. Merlin, S.R. Phillpot, J. Appl. Phys. 93 (2003) 793.

[2] X. Zhang, H.Q. Xie, M. Fujii, H. Ago, K. Takahashi, T. Ikuta, H. Abe, T. Shimizu, Appl. Phys. Lett. 86 (2005) 3.

[3] D.G. Cahill, K. Goodson, A. Majumdar, J. Heat Transfer-Trans. ASME 124 (2002) 223.

[4] X. Zheng, D.G. Cahill, P. Krasnochtchekov, R.S. Averback, J.C. Zhao, Acta Mater. 55 (2007) 5177.

[5] D.G. Cahill, Rev. Sci. Instrum. 61 (1990) 802.

[6] A. Majumdar, Annual Rev. Mater. Sci. 29 (1999) 505.

[7] F. Volklein, Thin Solid Films 188 (1990) 27.

[8] B.L. Zink, B. Revaz, J.J. Cherry, F. Hellman, Rev. Sci. Instrum. 76 (2005).

[9] L. La Spina, A.W. van Herwaarden, H. Schellevis, W.H.A. Wien, N. Nenadovic, L.K. Nanver, J. Microelectromechanical Syst. 16 (2007) 675.

[10] T. Tong, A. Majumdar, Rev. Sci. Instrum. 77 (2006).

[11] A. Jain, K.E. Goodson, J. Heat Transfer-Trans. ASME 130 (2008).

[12] M.Y. Efremov, E.A. Olson, M. Zhang, F. Schiettekatte, Z.S. Zhang, L.H. Allen, Rev. Sci. Instrum. 75 (2004) 179.

[13] E.A. Olson, M. Yu, Y. Efremov, M. Zhang, Z.S. Zhang, L.H. Allen, J. Microelectromechanical Syst. 12 (2003) 355. 
[14] M.Y. Efremov, F. Schiettekatte, M. Zhang, E.A. Olson, A.T. Kwan, R.S. Berry, L.H. Allen, Phys. Rev. Lett. 85 (2000) 3560.

[15] S.L. Lai, G. Ramanath, L.H. Allen, P. Infante, Z. Ma, Appl. Phys. Lett. 67 (1995) 1229.

[16] I.N. Sneddon, Fourier Transforms, McGraw-Hill, New York, 1951.

[17] Bateman Manuscript Project, H. Bateman, A. Erdélyi, U. S. Office of Naval Research, Tables of Integral Transforms, McGraw-Hill, New York, 1954.

[18] P.R. Bevington, D.K. Robinson, Data Reduction and Error Analysis for The Physical Sciences, McGraw-Hill, New York, 1992.

[19] CRC Handbook of Chemistry and Physics, CRC Press, Cleveland, Ohio, 1977.

[20] S.X. Cheng, P. Cebe, L.M. Hanssen, D.M. Riffe, A.J. Sievers, J. Opt. Soc. Am. B 4 (1987) 351.

[21] A. Jacquot, G. Chen, H. Scherrer, A. Dauscher, B. Lenoir, Sens. Actuators, A 117 (2005) 203.

[22] J.H. Lienhard IV, J.H. Lienhard V, A Heat Transfer Textbook, Phlogiston Press, Cambridge, Massachusetts, 2006, p. 528.

[23] C.H. Mastrangelo, Y.C. Tai, R.S. Muller, Sens. Actuators A21-A23 (1990), 856.

[24] J. Rupp, R. Birringer, Phys. Rev. B 36 (1987) 7888.

[25] J. Yu, Z.A. Tang, F.T. Zhang, H.T. Ding, Z.X. Huang, J. Heat Transfer-Trans. ASME 132 (2010).

[26] P.J. McCluskey, J.J. Vlassak, in: A. Misra, J.P. Sullivan, H. Huang, K. Lu, S. Asif (Eds.), Mechanics of Nanoscale Materials and Devices, San Francisco, U.S.A., 
April 17-21, 2006, Materials Research Society Symposium Proceedings 924E (2006) 0924-Z08-14.

[27] T.Q. Qiu, C.L. Tien, J. Heat Transfer-Trans. ASME 115 (1993) 842.

[28] S. Kumar, G.C. Vradis, J. Heat Transfer-Trans. ASME 116 (1994) 28.

\section{Tables}

Table 1: Approximate dimensions of nTTA components

Table 2: FEM input materials properties [19-23]

Table 3: FEM measurement simulation results

Table 4: Applied current in milli-Amperes for a given cell and measured quantity

\section{Figures}

Figure 1: nano-thermal transport array schematic.

Figure 2: Thermal cell schematic: (a) Plan view, and (b) Cross-section view.

Figure 3: Schematic of control volume in relation to the entire thermal cell.

Figure 4: FEM average node temperature distribution along $y$-direction for a bare thermal cell and cell with $50 \mathrm{~nm} \mathrm{Cu}$ sample, simulated for $5 \mathrm{~ms}$ with respective currents of 5 and $10 \mathrm{~mA}$.

Figure 5: FEM average temperature history of control volume nodes. Data is shown as dashes and the $5^{\text {th }}$ order and $7^{\text {th }}$ order polynomial fits are shown for the bare cell and the cell with $50 \mathrm{~nm} \mathrm{Cu}$, respectively.

Figure 6: Radiation from $\mathrm{CV}$ and membrane relative to the power dissipated in the thermistor for the bare cell.

Figure 7: Results of the analysis of the FEM results (data points with error bars) compared to input values (solid line): heat capacity of the CV of a bare cell (a), effusivity 
of a bare-cell membrane (b), heat capacity of the $\mathrm{CV}$ of a cell with a $50 \mathrm{~nm} \mathrm{Cu}$ coating (c), effusivity of a membrane with a $50 \mathrm{~nm} \mathrm{Cu}$ coating (d).

Figure 8: Emissivity calculated from the FEM simulations along with FEM input value. The error on the calculated emissivity is large at low temperatures, but the emissivity quickly converges to the input value at elevated temperatures where radiation is a more important heat loss mechanism.

Figure 9: nTTA fabrication flow. One thermal cell is shown for clarity. Detailed step-bystep procedures can be found in the text of the paper.

Figure 10: Schematic of the experimental setup.

Figure 11: Raw voltage data recorded from a $5 \mathrm{~mA}, 20 \mathrm{~ms}$ current pulse across the $100 \Omega$ precision resistor and a narrow thermistor.

Figure 12: Thickness of the copper sample multilayers.

Figure 13: Typical temperature history for a bare cell and cell with $61 \mathrm{~nm}$ sample (a). Typical heating rate for bare cell and cell with $61 \mathrm{~nm}$ sample (b).

Figure 14: Typical fit to the power data for a bare cell and a cell with a $61 \mathrm{~nm}$ copper film.

Figure 15: Typical thermal parameter fit results for bare cell (a) and cell with $61 \mathrm{~nm} \mathrm{Cu}$ (b) heat capacity; effusivity results for the same cases (c).

Figure 16: Heat capacity (a) and effusivity (b) results for cells with and without copper samples.

Figure 17: Calculated thermal conductivity of thin copper films from measured thermal parameters, with $k_{S i N}=3.2 \mathrm{~W} / \mathrm{m} \mathrm{K}$ and $\left(\rho c_{P}\right)_{S i N}=2.1 \mathrm{MJ} / \mathrm{m}^{3} \mathrm{~K}$ [23].

Figure 18: TEM cross-section of a 3-layer $\mathrm{Cu}$ film showing scattering interfaces.

Figure 19: Normalized thermal conductivity measurements of thin copper multilayer films, compared to Qiu's thermal conductivity model [27] for multilayer copper films.

Figure B1: Relative errors of measured thermal parameters as a function of the value of these thermal parameters, (a) relative error of effusivity and (b) relative error of heat 
capacity. Data reduction used a moving $0.3 \mathrm{~ms}$ time-span for the heating rate fit and a 4 ms time-span to fit a $5^{\text {th }}$ order polynomial to the temperature history.

\section{Tables}

Table 1: Approximate dimensions of nTTA components.

\begin{tabular}{|l|c|c|c|}
\hline & Length (x) & Width (y) & Thickness (z) \\
\hline Substrate $(\mathrm{Si})$ & $55 \mathrm{~mm}$ & $55 \mathrm{~mm}$ & $0.7 \mathrm{~mm}$ \\
\hline Membrane $\left(\mathrm{Si}_{3} \mathrm{~N}_{4}\right)$ & $5 \mathrm{~mm}$ & $3 \mathrm{~mm}$ & $80 \mathrm{~nm}$ \\
\hline Narrow thermistor $(\mathrm{W})$ & $3 \mathrm{~mm}$ & $84 \mu \mathrm{m}$ & $125 \mathrm{~nm}$ \\
\hline Wide thermistor $(\mathrm{W})$ & $3 \mathrm{~mm}$ & $800 \mu \mathrm{m}$ & $125 \mathrm{~nm}$ \\
\hline
\end{tabular}

Table 2: FEM input materials properties [18-23].

\begin{tabular}{|l|c|c|c|c|}
\hline & $k(\mathrm{~W} / \mathrm{m} \mathrm{K})$ & $\rho\left(\mathrm{kg} / \mathrm{m}^{3}\right)$ & $c_{P}(\mathrm{~J} / \mathrm{kg} \mathrm{K})$ & $\varepsilon$ \\
\hline Thermistor $(\mathrm{W})$ & 174 & 19300 & 132 & 0.02 \\
\hline Membrane $\left(\mathrm{Si}_{3} \mathrm{~N}_{4}\right)$ & $2.3^{*}$ & 3000 & 700 & 0.18 \\
\hline Sample $(\mathrm{Cu})$ & 401 & 8960 & 384 & 0.01 \\
\hline
\end{tabular}

* Value is from a $\mathrm{SiN}_{\mathrm{X}} / \mathrm{SiO}_{2} / \mathrm{SiN}_{\mathrm{X}}$ film stack.

Table 3: FEM measurement simulation results.

\begin{tabular}{|l|c|c|c|c|}
\hline & Parameter & Input & Result & Error (\%) \\
\hline \multirow{2}{*}{ Bare cell } & $C_{P}(\mathrm{~nJ} / \mathrm{K})$ & 134.8 & 133.4 & 1.1 \\
\cline { 2 - 5 } & $\phi\left(\mathrm{J} / \mathrm{m}^{2} \mathrm{~s}^{1 / 2} \mathrm{~K}\right)$ & 2198 & 2257 & 2.7 \\
\hline $\begin{array}{l}\text { Cell with } 50 \mathrm{~nm} \\
\text { Cu coating }\end{array}$ & $C_{P}(\mathrm{~nJ} / \mathrm{K})$ & 198 & 192 & 3.1 \\
\cline { 2 - 5 } & $\phi\left(\mathrm{J} / \mathrm{m}^{2} \mathrm{~s}^{1 / 2} \mathrm{~K}\right)$ & 21820 & 21480 & 1.5 \\
\hline
\end{tabular}


Table 4: Applied current in milli-Amperes for a given cell and measured quantity.

\begin{tabular}{|l|c|c|c|c|c|}
\hline & Cells 1-5 & Cells 6-10 & Cells 11-15 & Cells 16-20 & Cells 21-25 \\
\hline$R_{0}$ & 1 & 1 & 1 & 1 & 8 \\
\hline$\phi_{M}, C_{P}$ (cell) & 5 & 5 & 5 & 5 & 80 \\
\hline$\phi_{M}$ (sample) & 12 & 10 & 8 & 6 & - \\
\hline$C_{P}$ (sample) & 24 & 20 & 16 & 12 & $80-90$ \\
\hline$\varepsilon_{z}$ (cell) & - & - & - & $11-12$ & 80 \\
\hline$\varepsilon_{z}$ (sample) & - & - & - & $14.5-18$ & $80-90$ \\
\hline
\end{tabular}




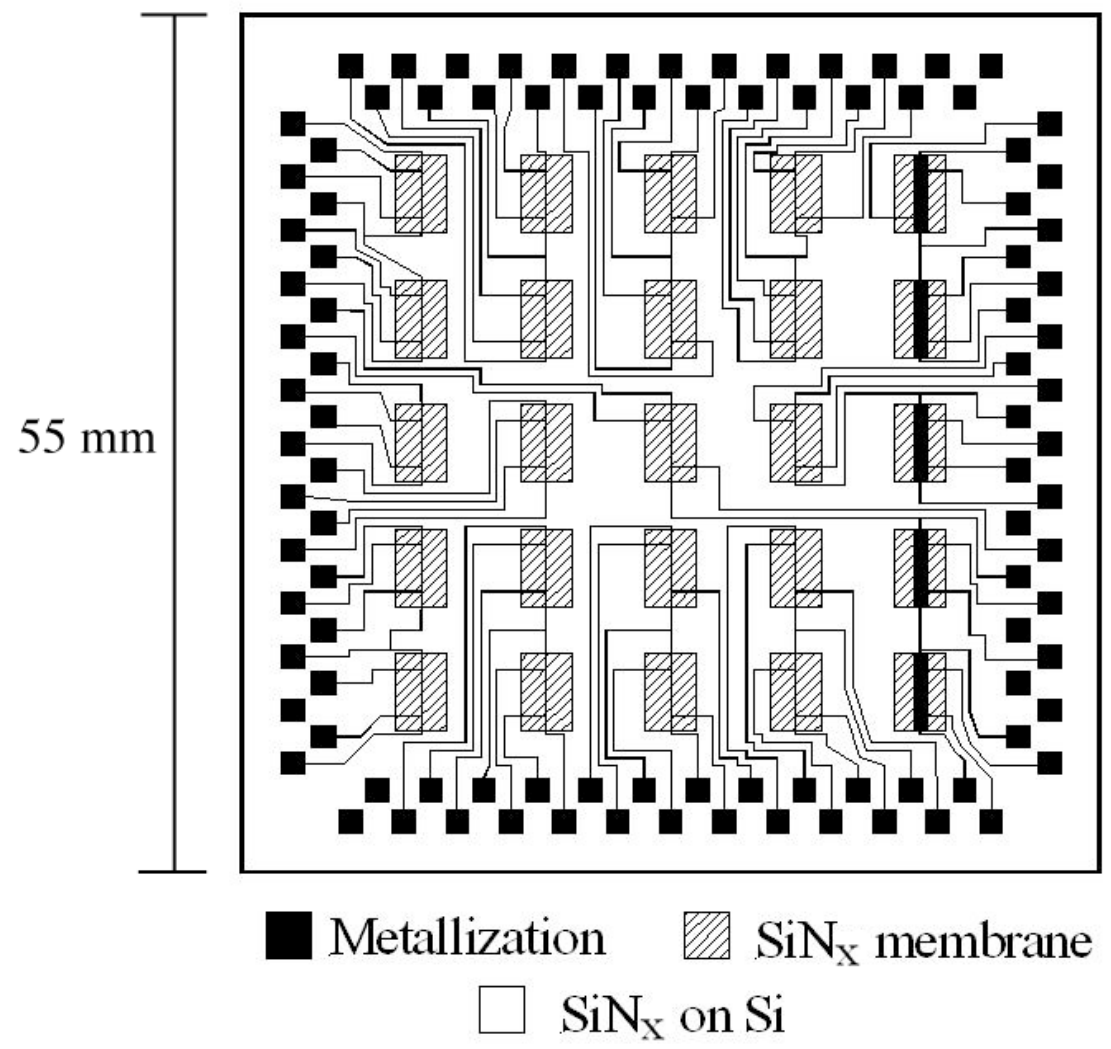

Figure 1: nano-thermal transport array schematic 


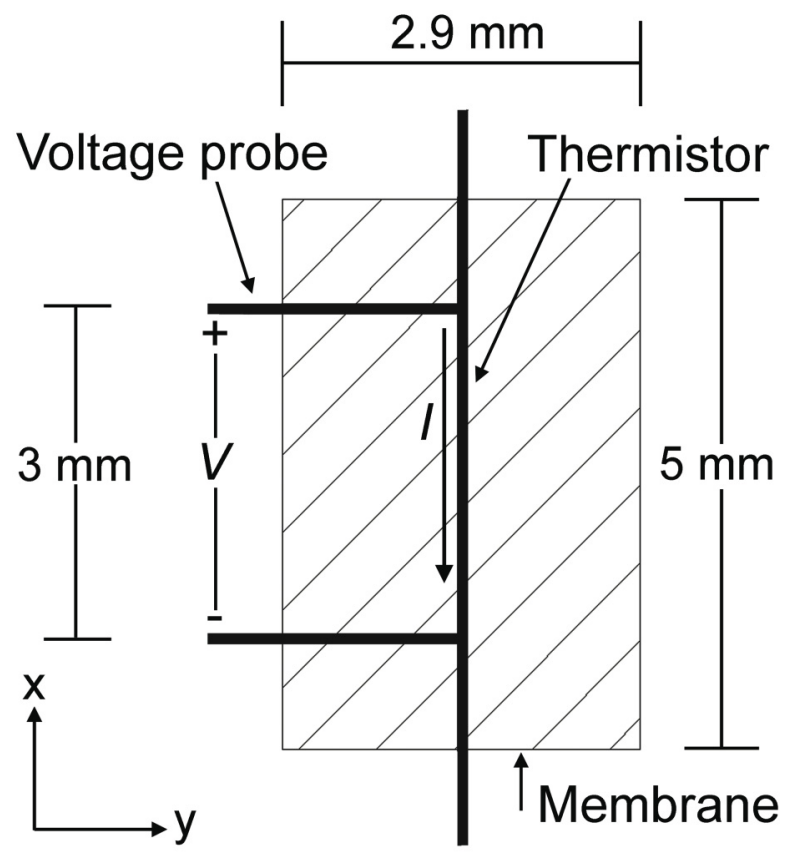

(a)
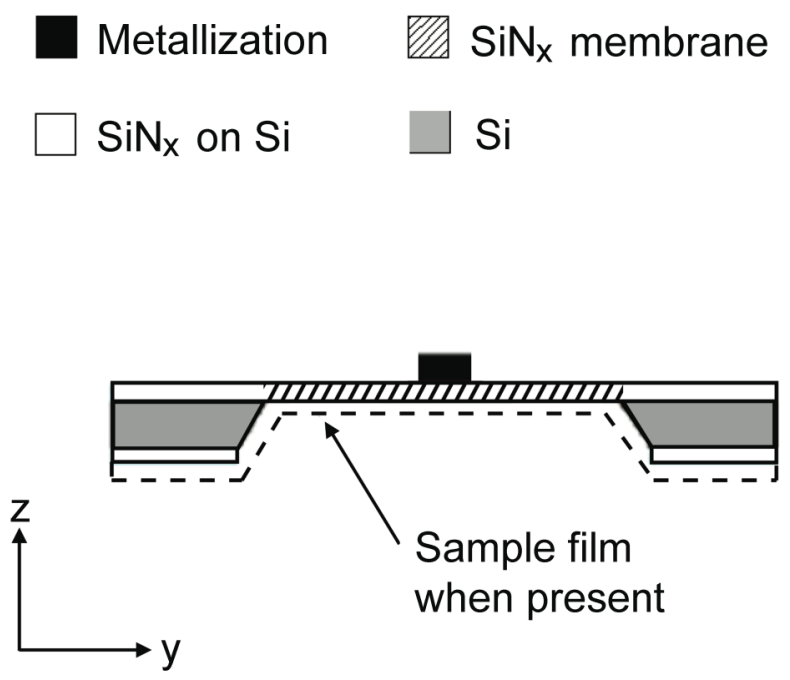

(b)

Figure 2: Thermal cell schematic: (a) Plan view, and (b) Cross-section view. 


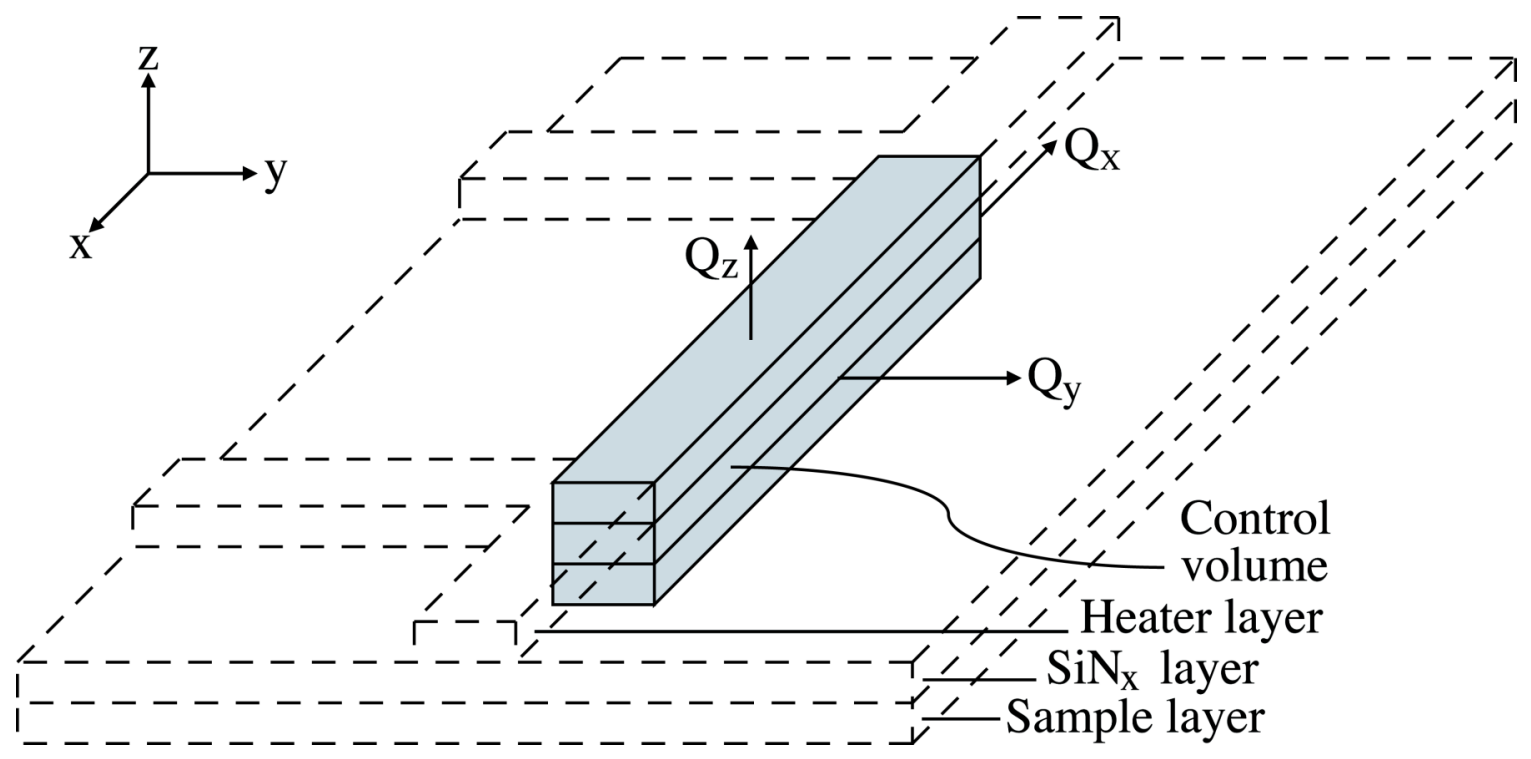

Figure 3: Schematic of control volume CV in relation to the entire thermal cell 


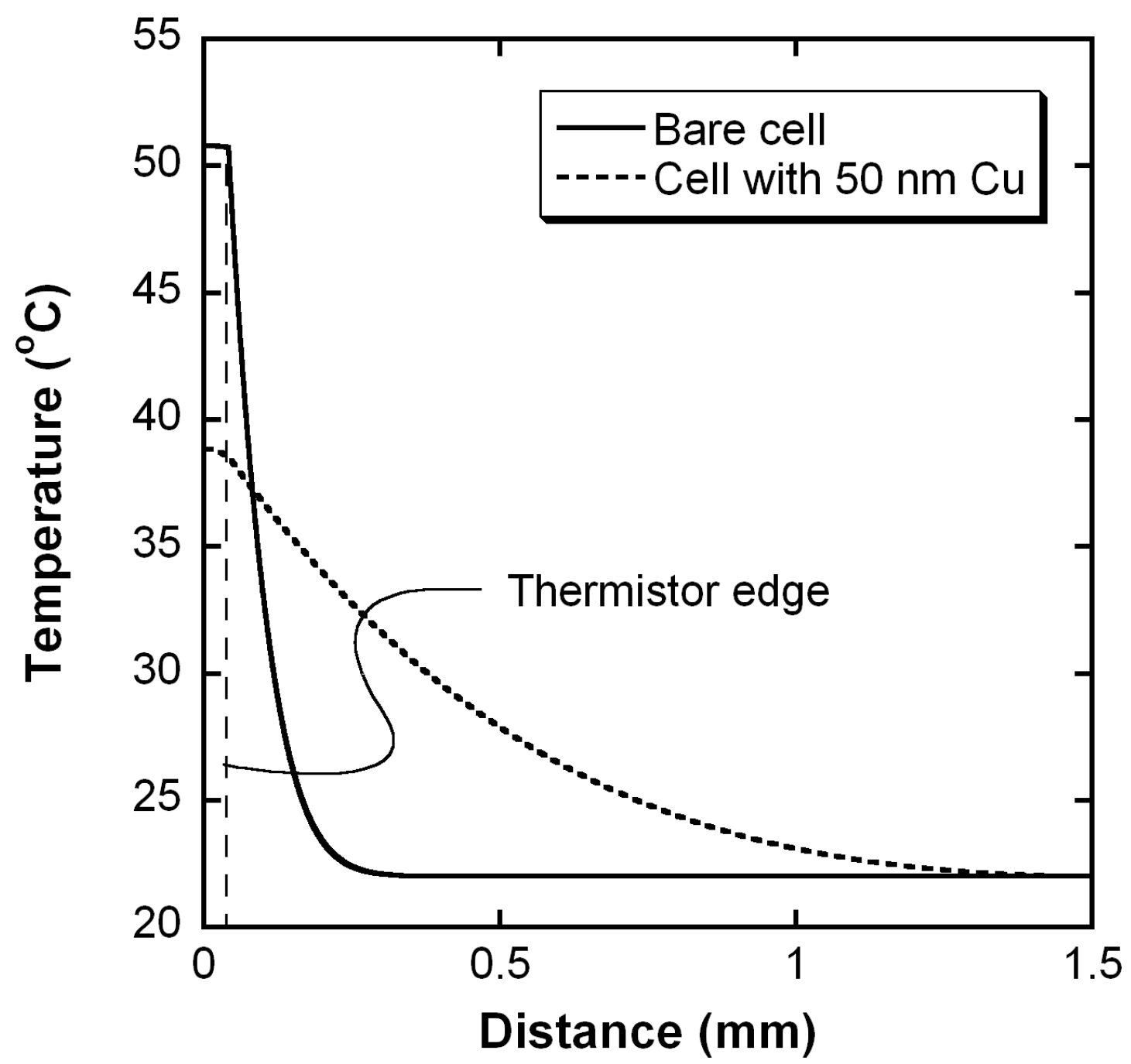

Figure 4: FEM average node temperature distribution along $y$-direction for a bare thermal cell and cell with $50 \mathrm{~nm} \mathrm{Cu}$ sample, simulated for $5 \mathrm{~ms}$ with respective currents of 5 and $10 \mathrm{~mA}$. 


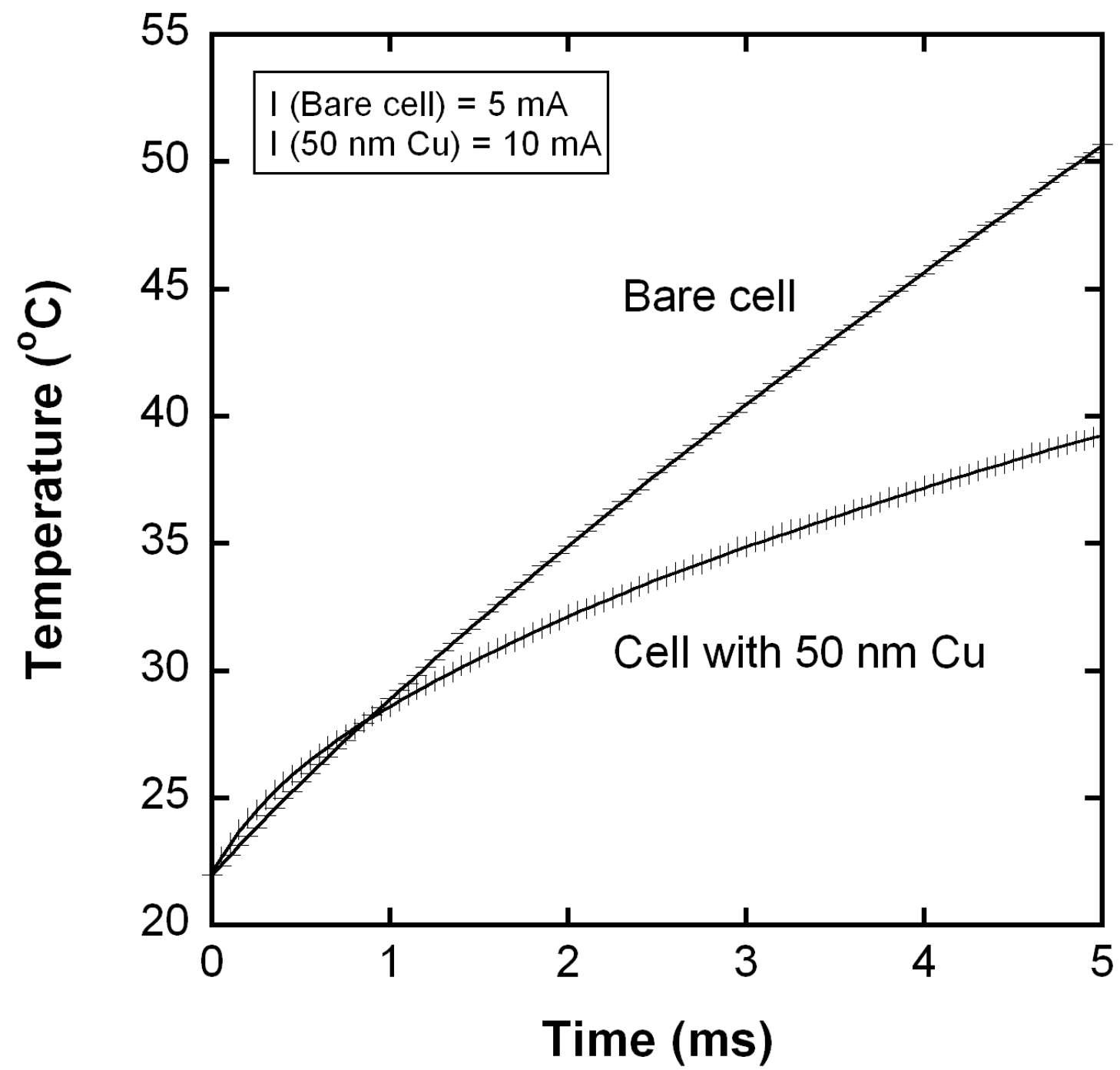

Figure 5: FEM average temperature history of control volume nodes. Data is shown as dashes and the $5^{\text {th }}$ order and $7^{\text {th }}$ order polynomial fits are shown for the bare cell and the cell with $50 \mathrm{~nm} \mathrm{Cu}$, respectively. 


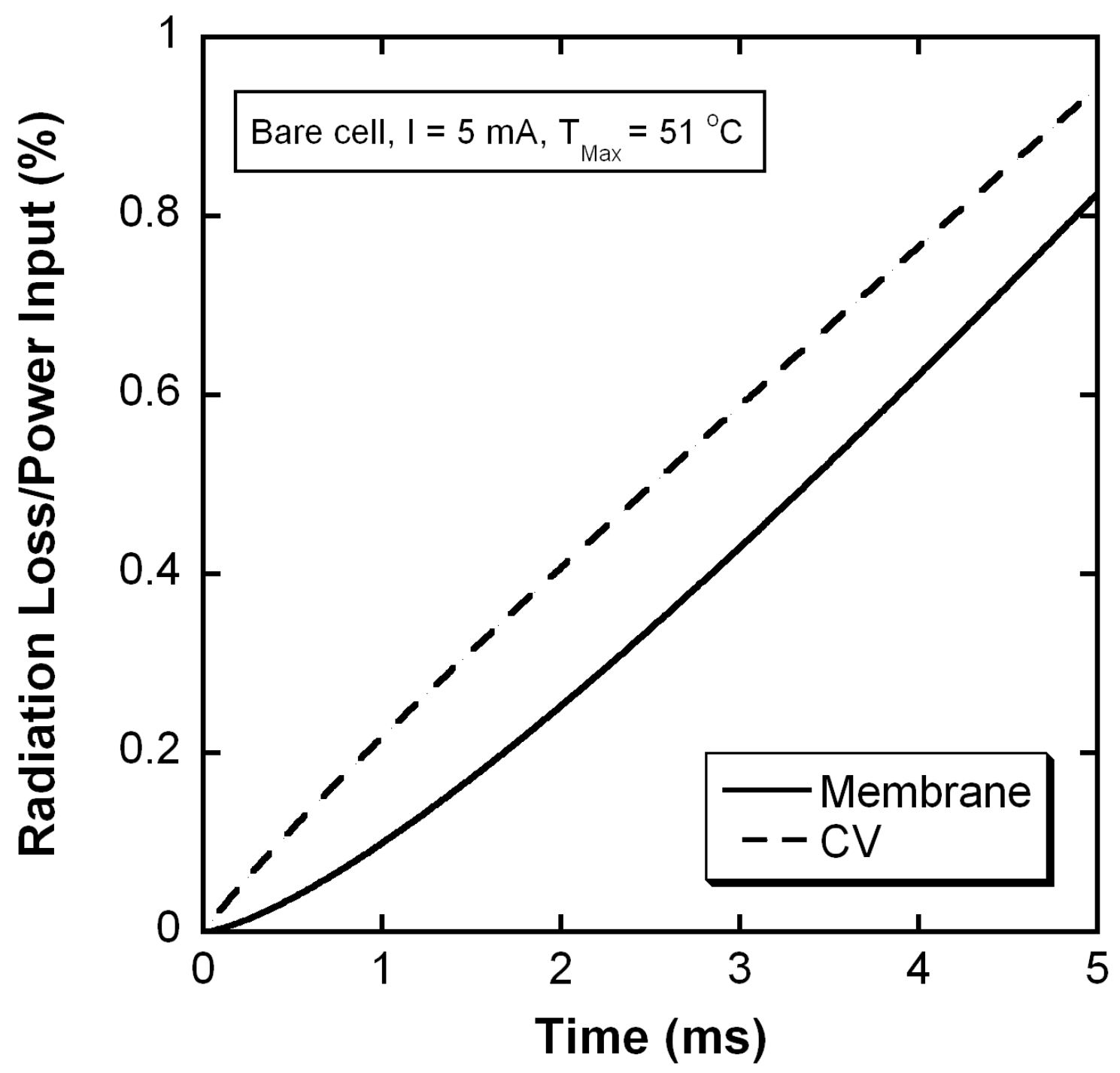

Figure 6: Radiation from control volume and membrane relative to the power dissipated in the thermistor for the bare cell. 

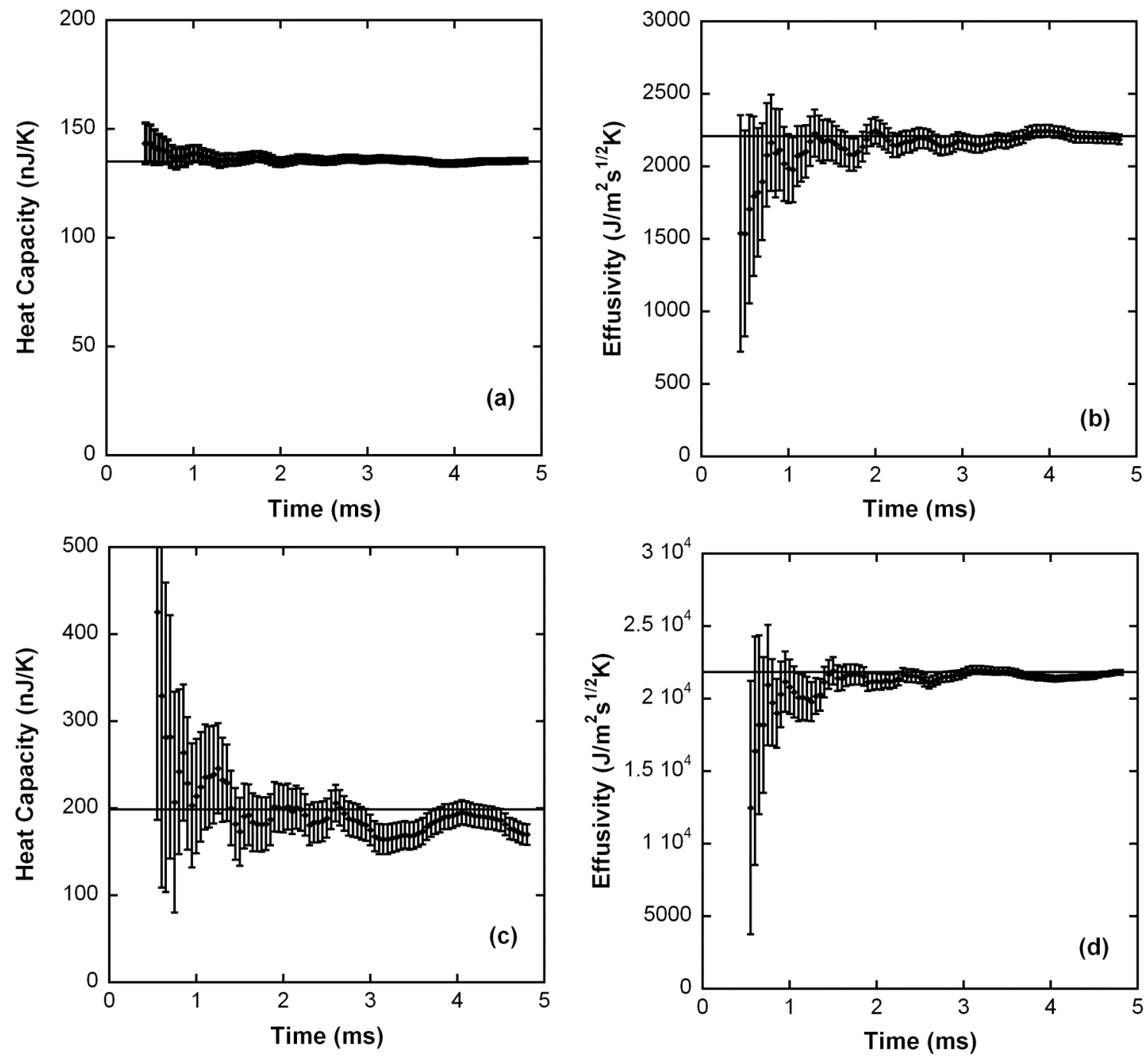

Figure 7: Results of the analysis of the FEM results (data points with error bars) compared to input values (solid line): heat capacity of the CV of a bare cell (a), effusivity of a bare-cell membrane (b), heat capacity of the $\mathrm{CV}$ of a cell with a $50 \mathrm{~nm} \mathrm{Cu}$ coating (c), effusivity of a membrane with a $50 \mathrm{~nm} \mathrm{Cu}$ coating (d). 


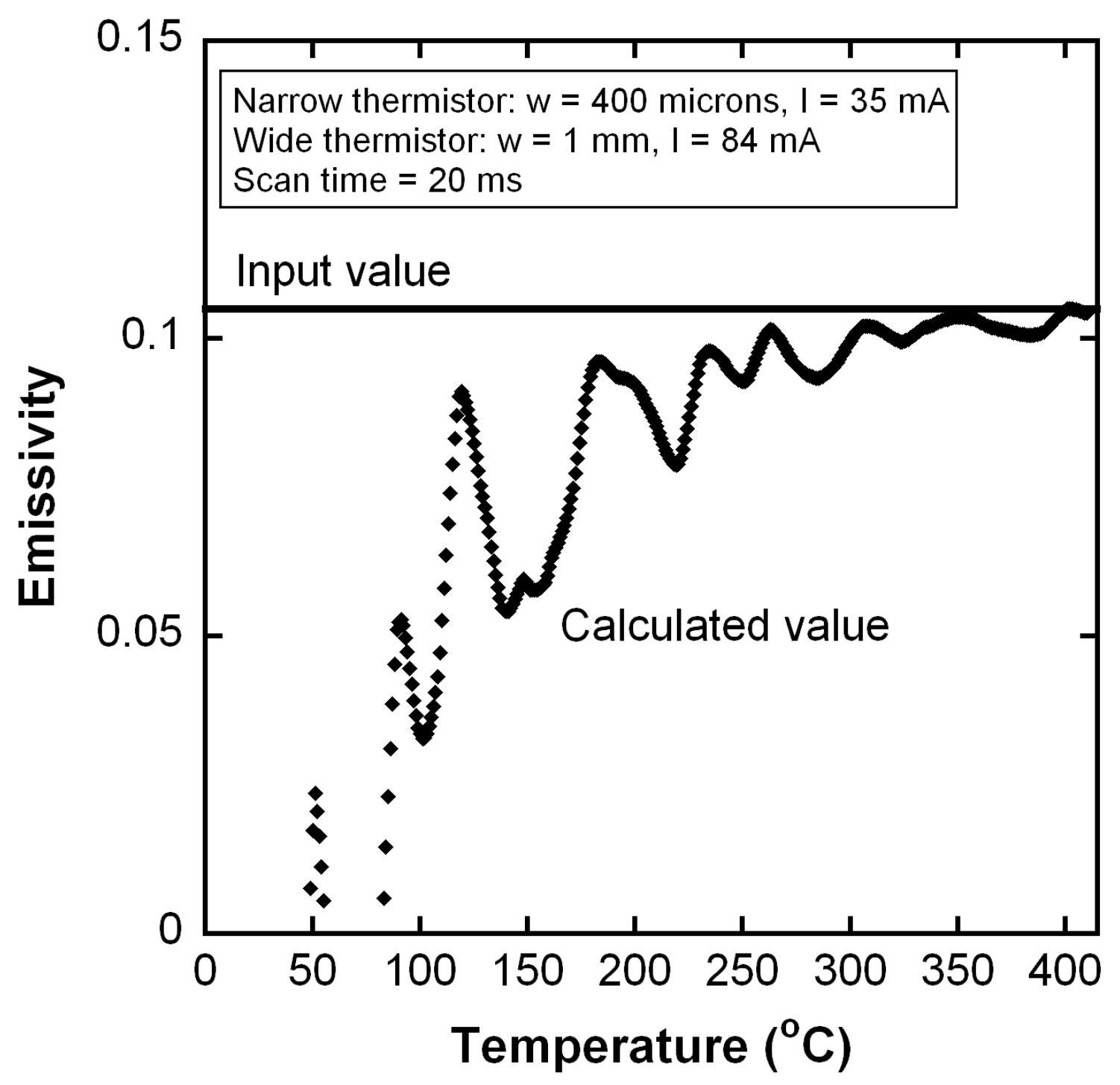

Figure 8: Emissivity calculated from the FEM simulations along with FEM input value. The error on the calculated emissivity is large at low temperatures, but the emissivity quickly converges to the input value at elevated temperatures where radiation is a more important heat loss mechanism. 


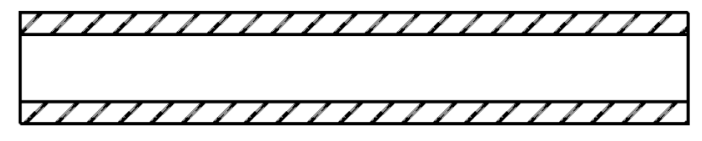

(a)

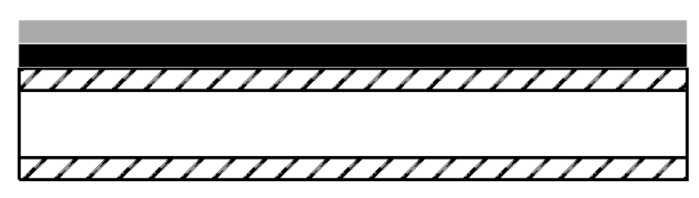

(b)

Exos

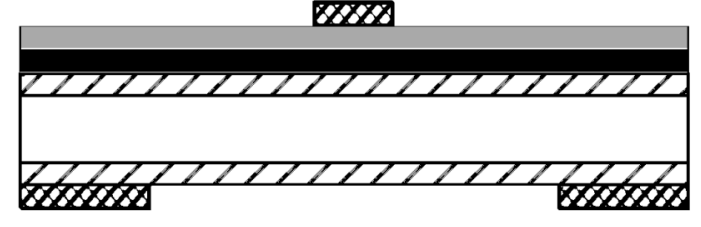

(c)

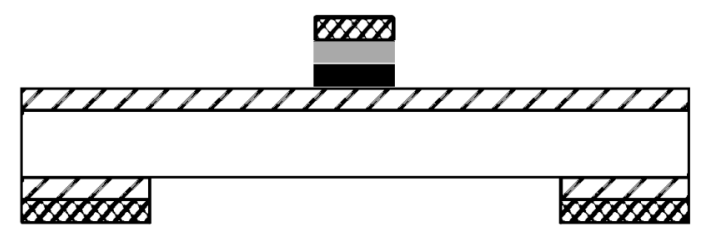

(d)

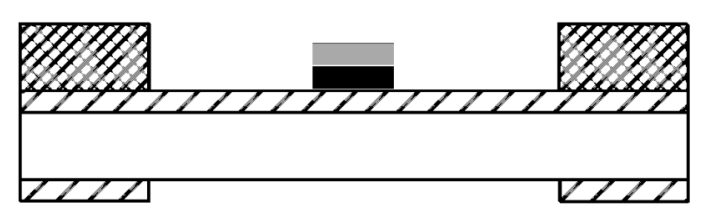

(e)

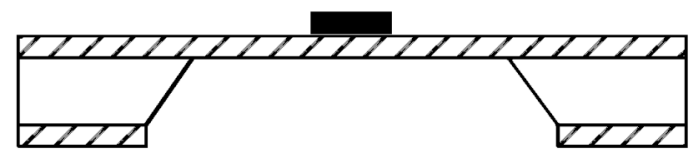

(f)
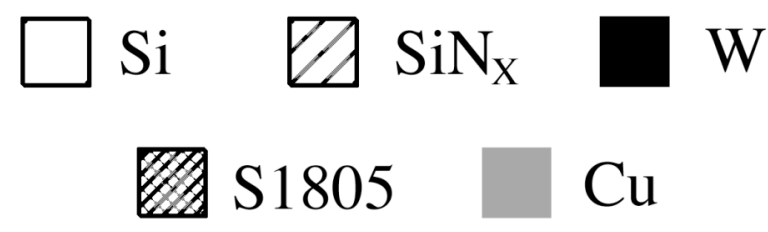

Figure 9: nTTA fabrication flow. One thermal cell is shown for clarity. Detailed step-bystep procedures can be found in the text of the paper. 


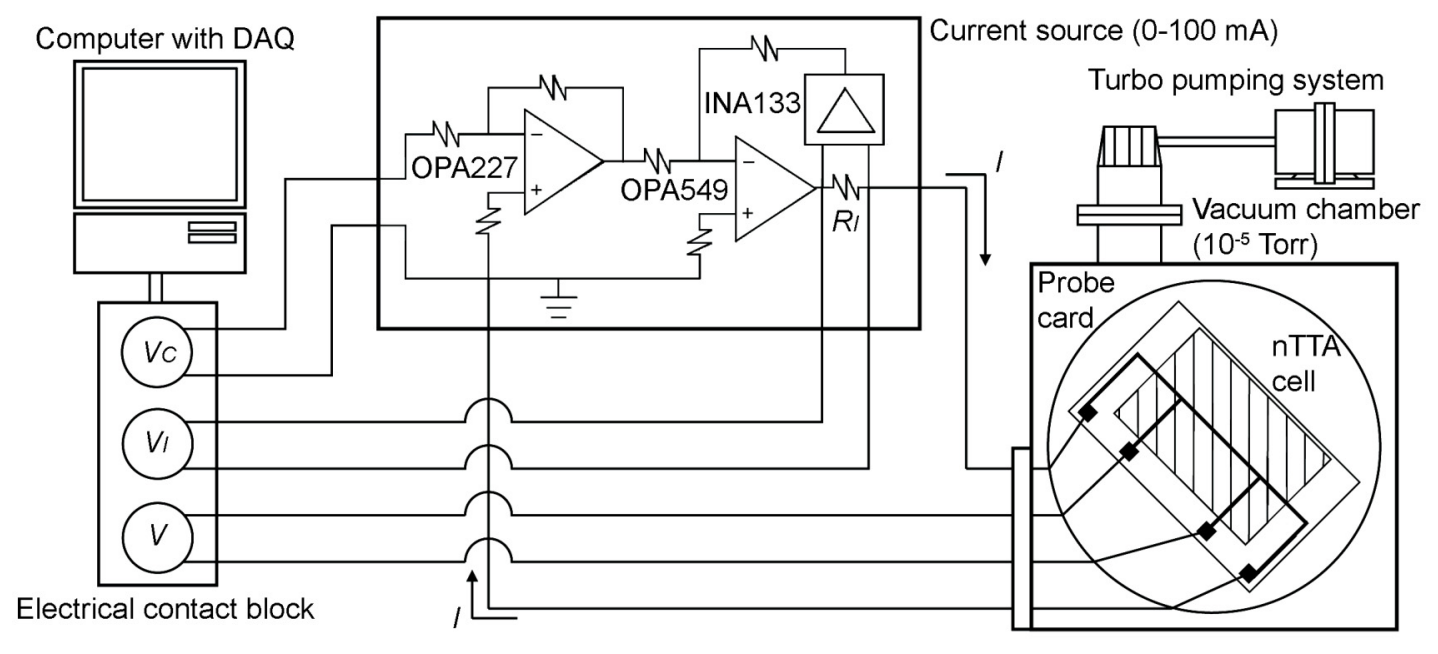

Figure 10: Schematic of the experimental setup 


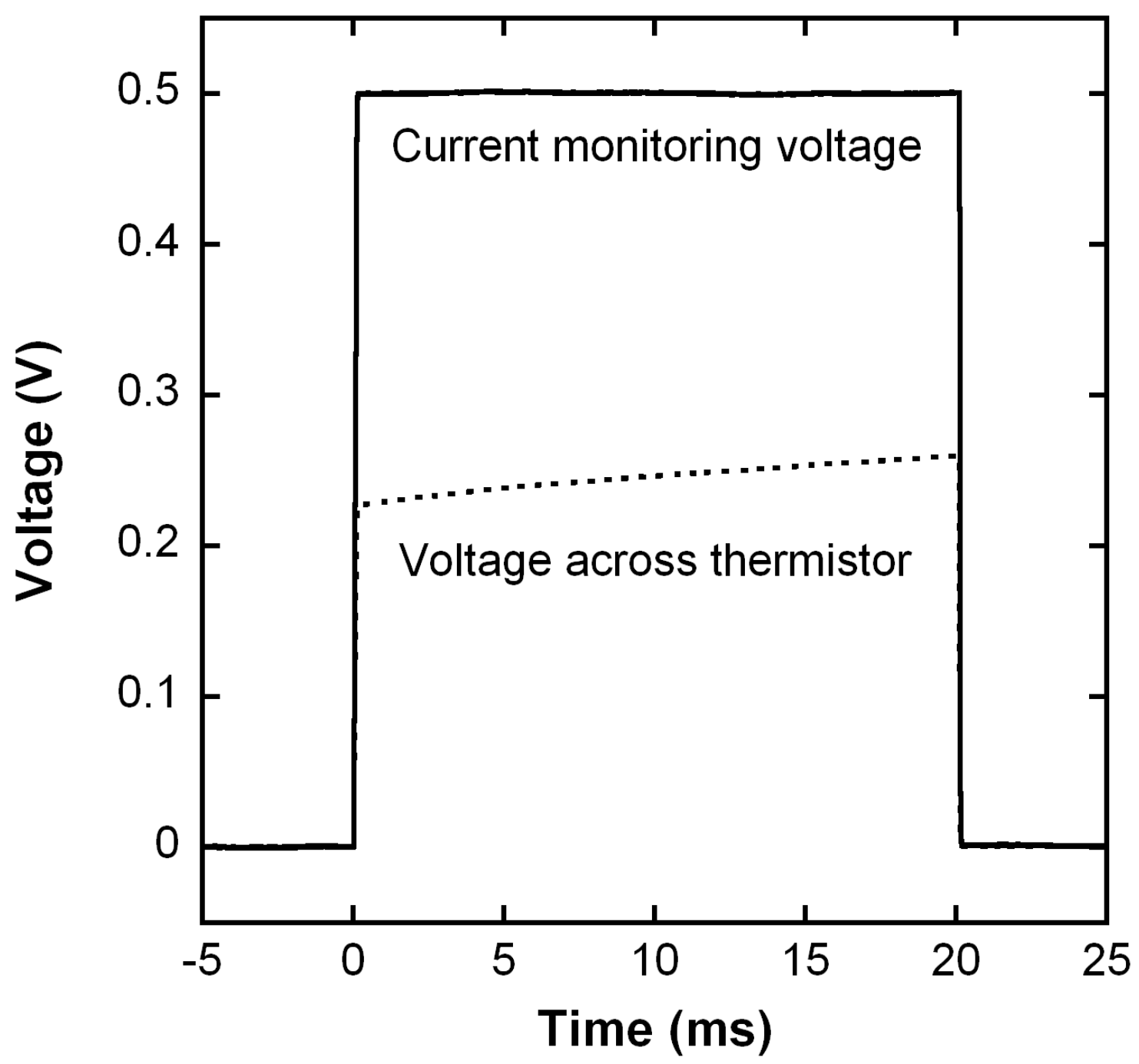

Figure 11: Raw voltage data recorded from a $5 \mathrm{~mA}, 20 \mathrm{~ms}$ current pulse across the $100 \Omega$ precision resistor and a narrow thermistor. 


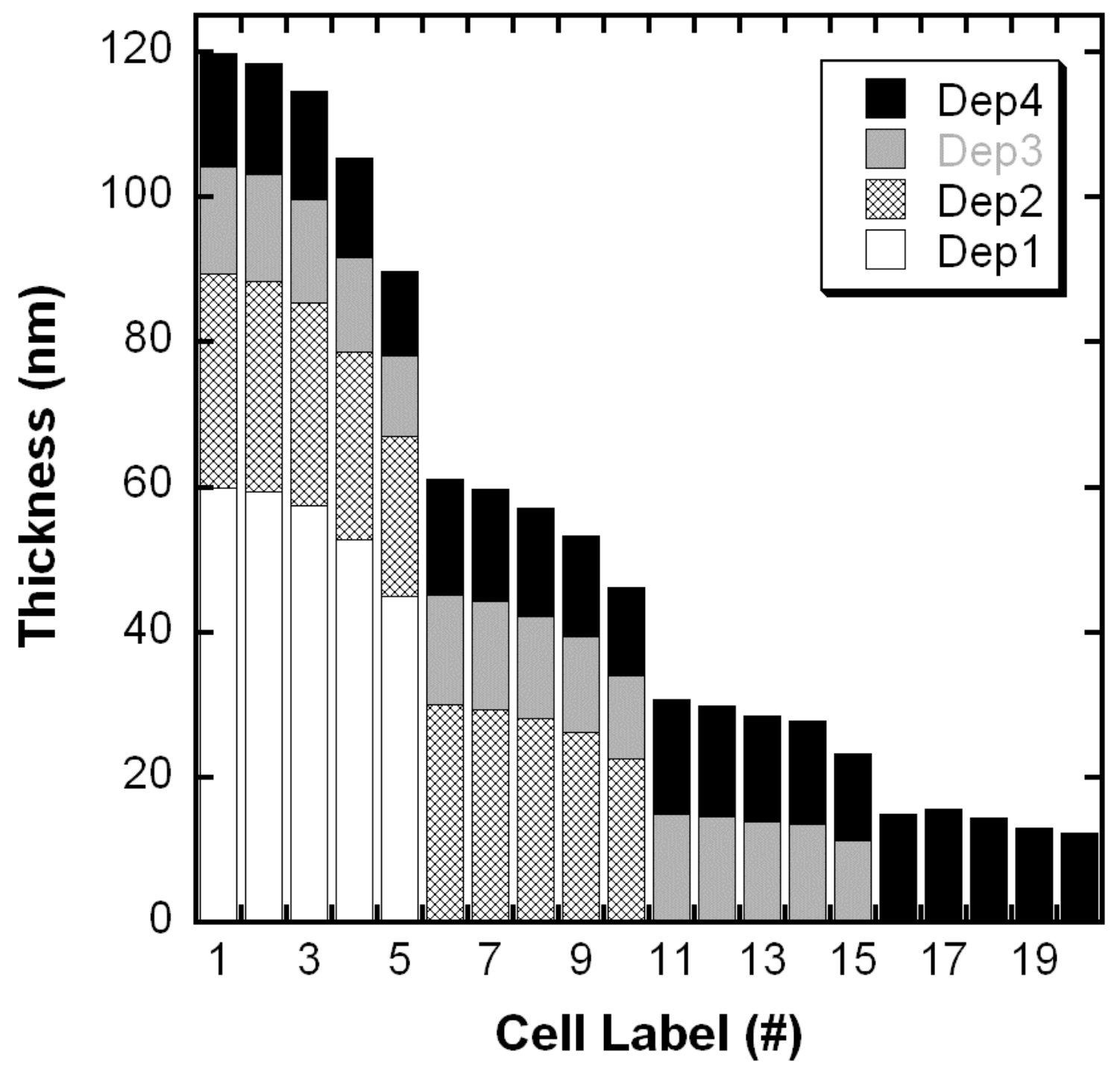

Figure 12: Thickness of the copper sample multilayers. 


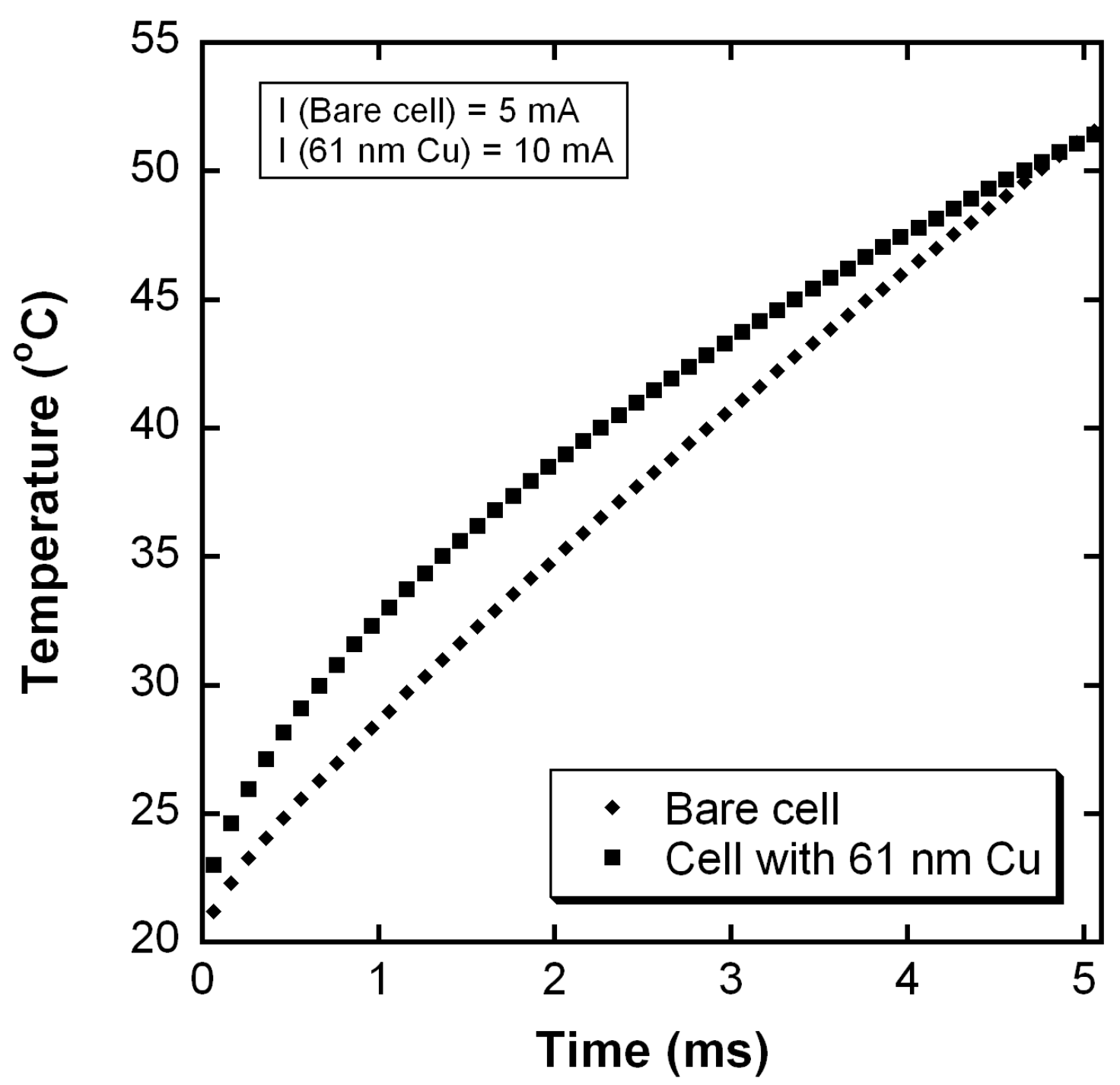

(a) 


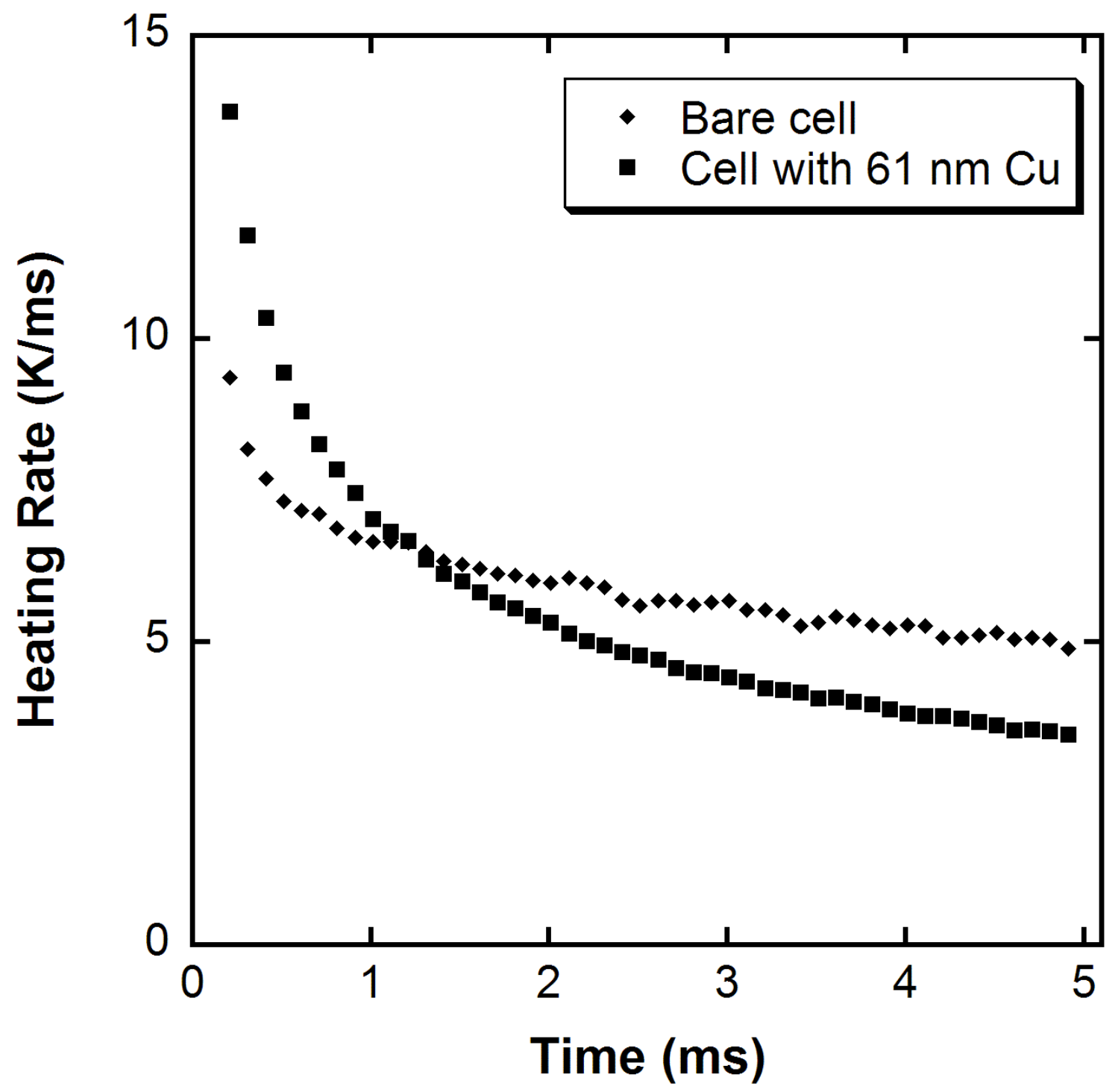

(b)

Figure 13: Typical temperature history for a bare cell and cell with $61 \mathrm{~nm}$ sample (a). Typical heating rate for bare cell and cell with $61 \mathrm{~nm}$ sample (b). 


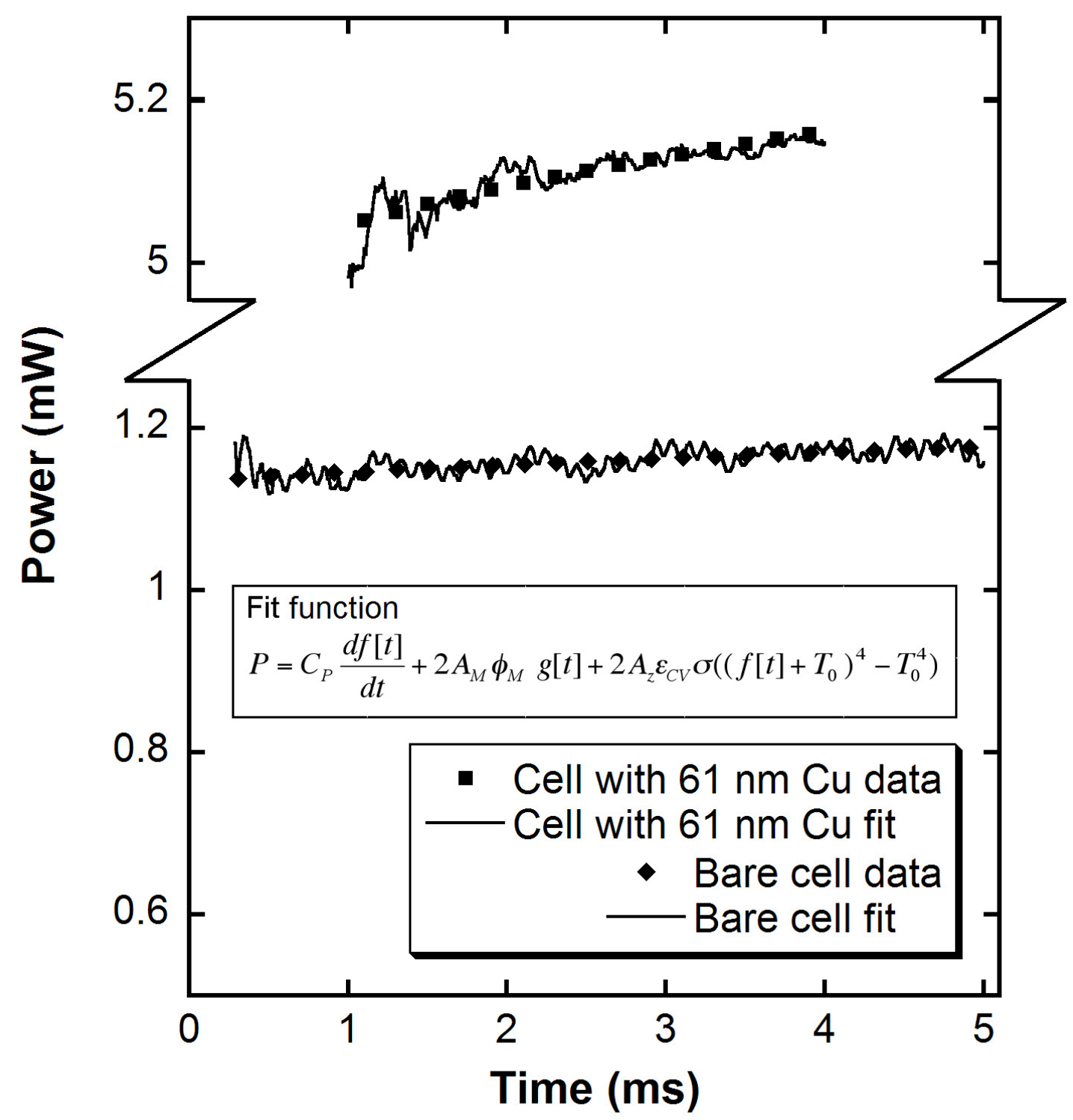

Figure 14: Typical fit to the power data for a bare cell and a cell with a $61 \mathrm{~nm}$ copper film. 


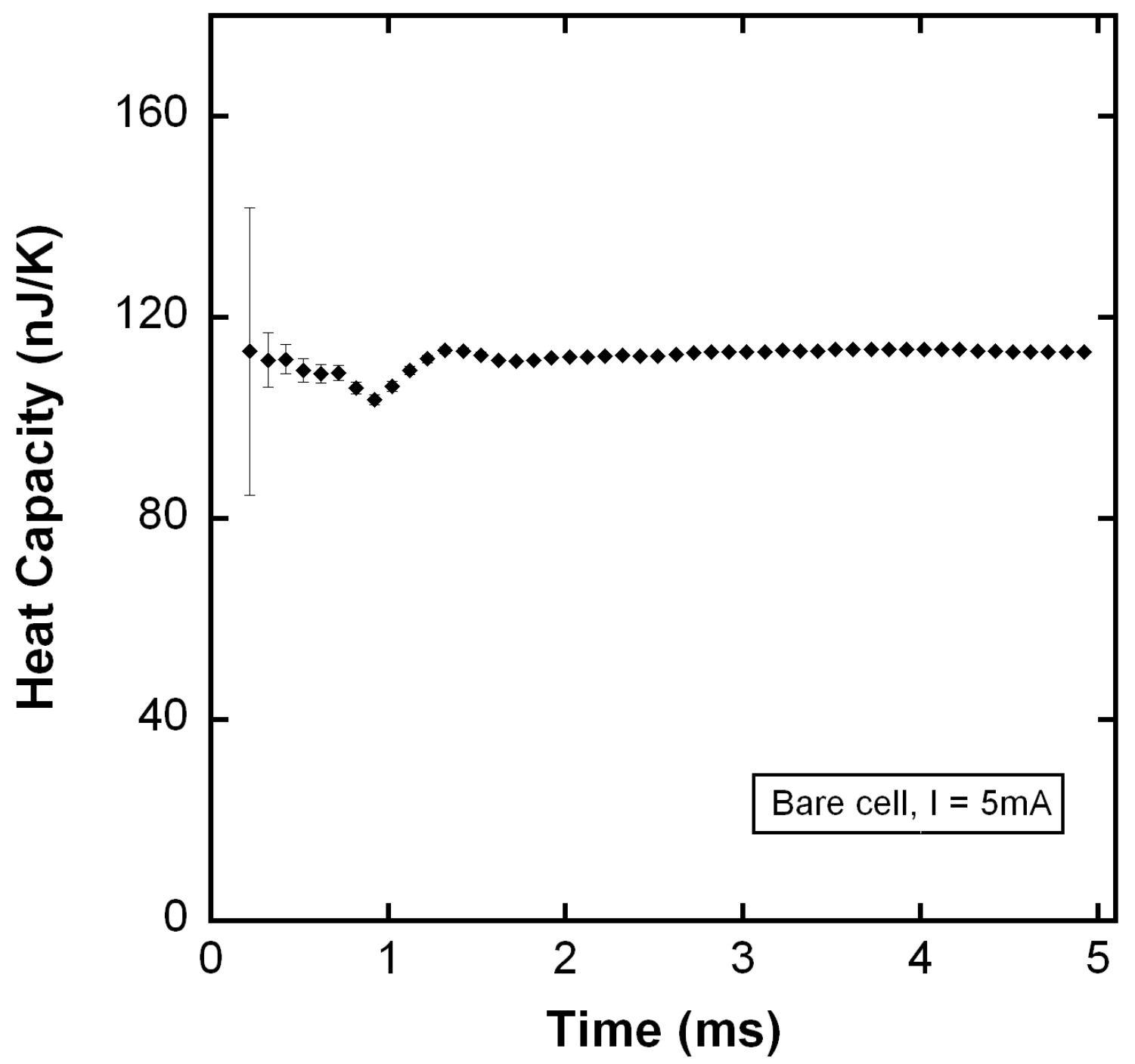

(a) 


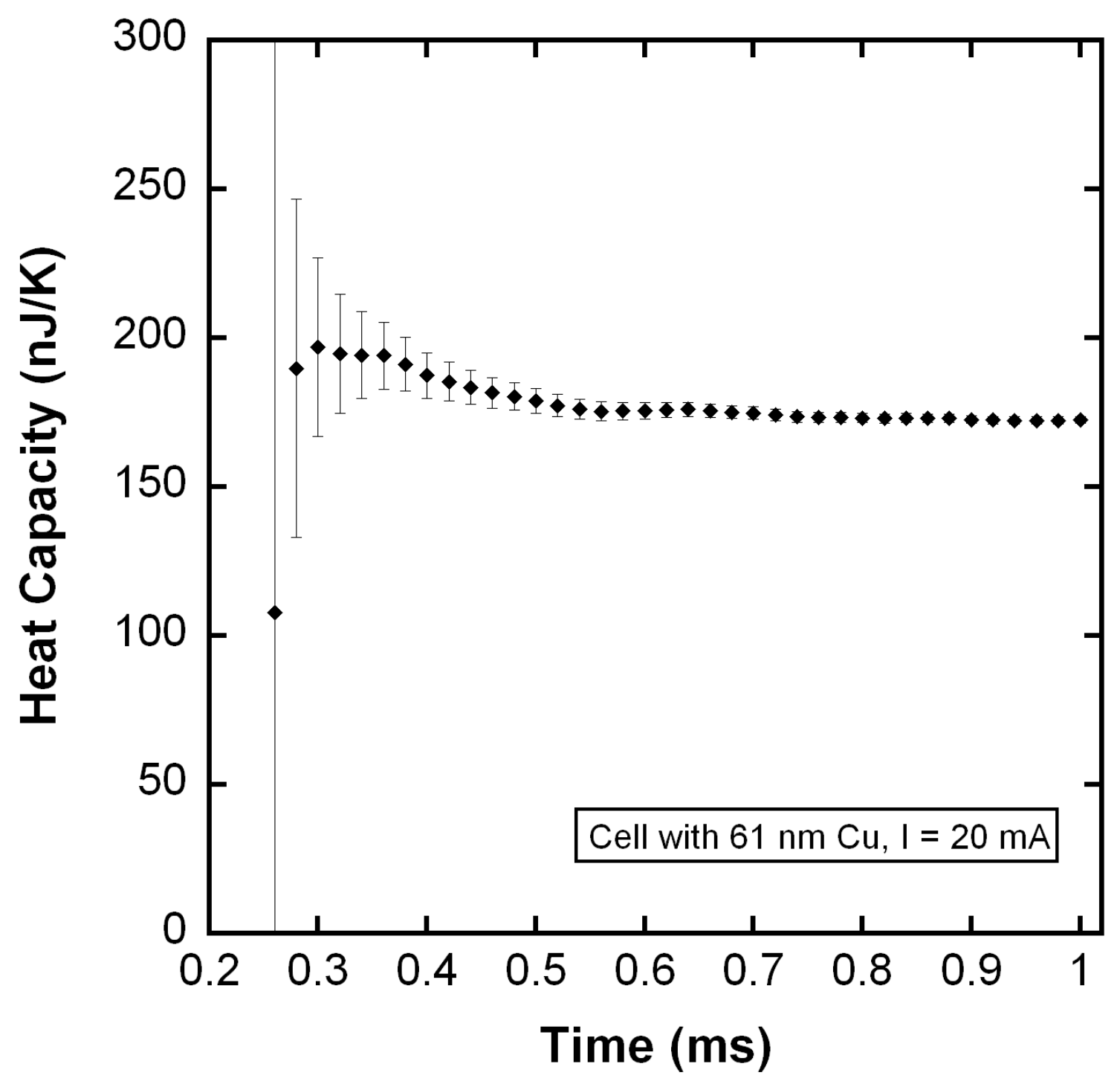

(b) 


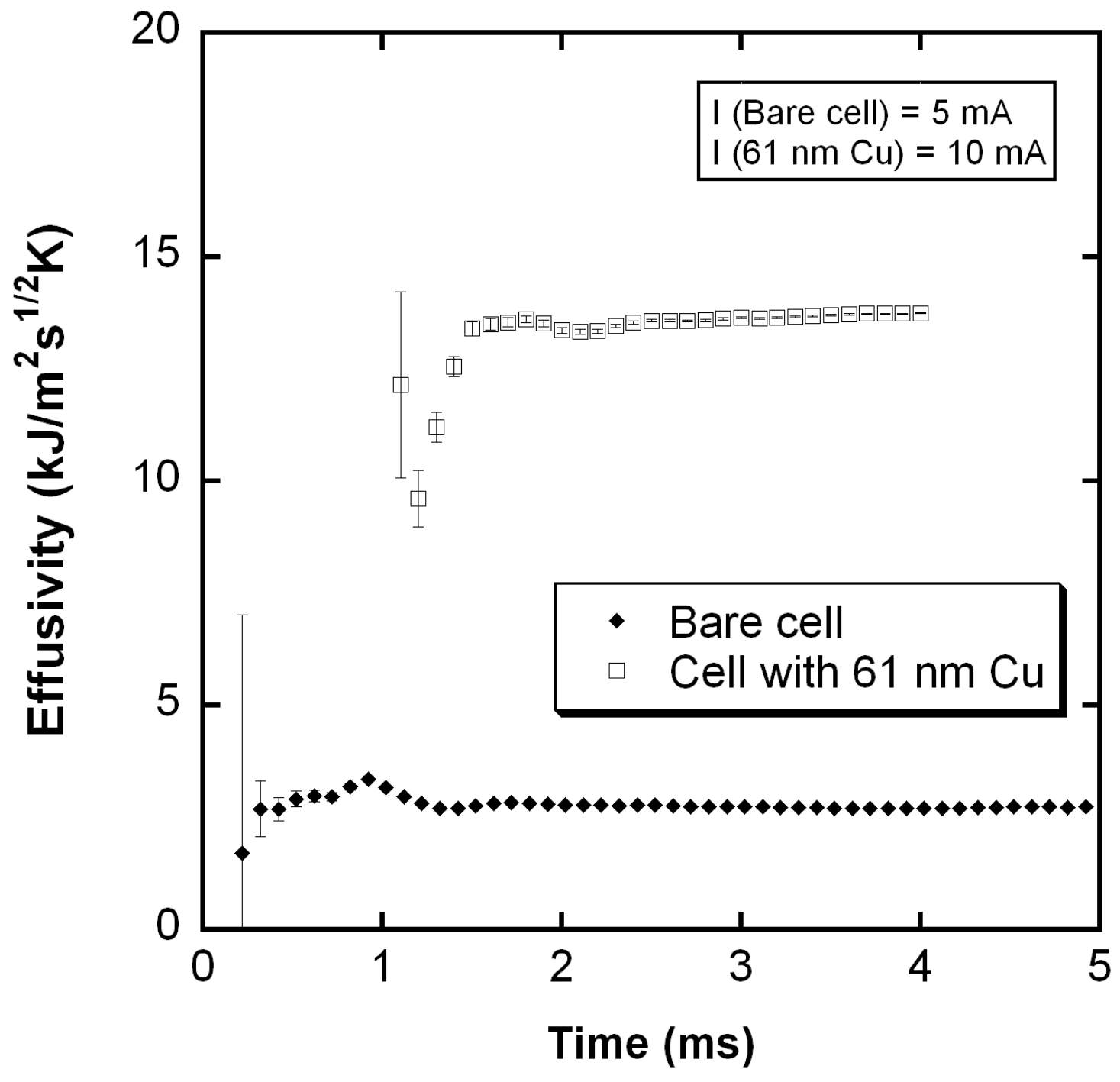

(c)

Figure 15: Typical thermal parameter fit results for bare cell (a) and cell with $61 \mathrm{~nm} \mathrm{Cu}$ (b) heat capacity; effusivity results for the same cases (c). 


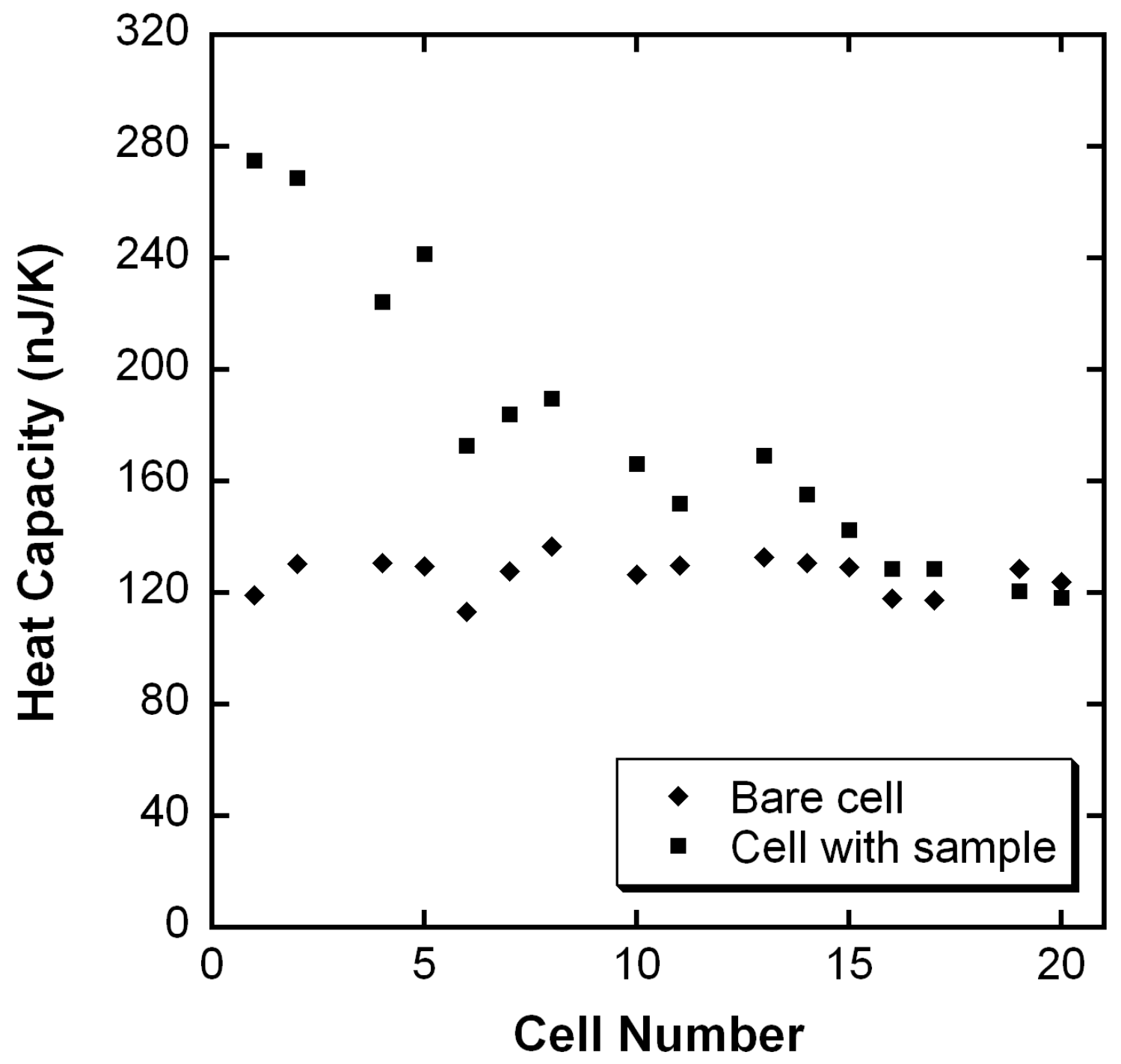

(a) 


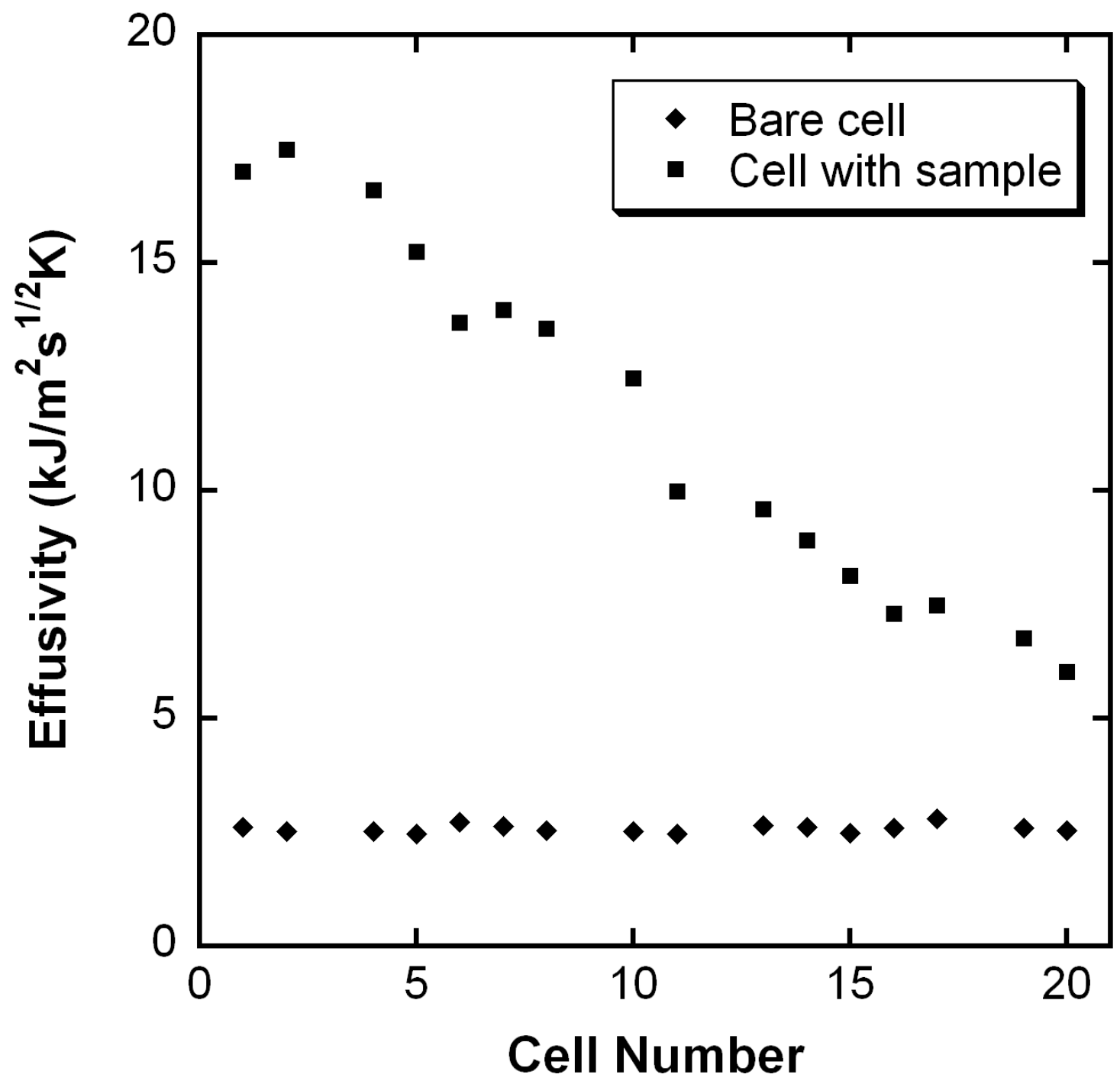

(b)

Figure 16: Heat capacity (a) and effusivity (b) results for cells with and without copper samples. 


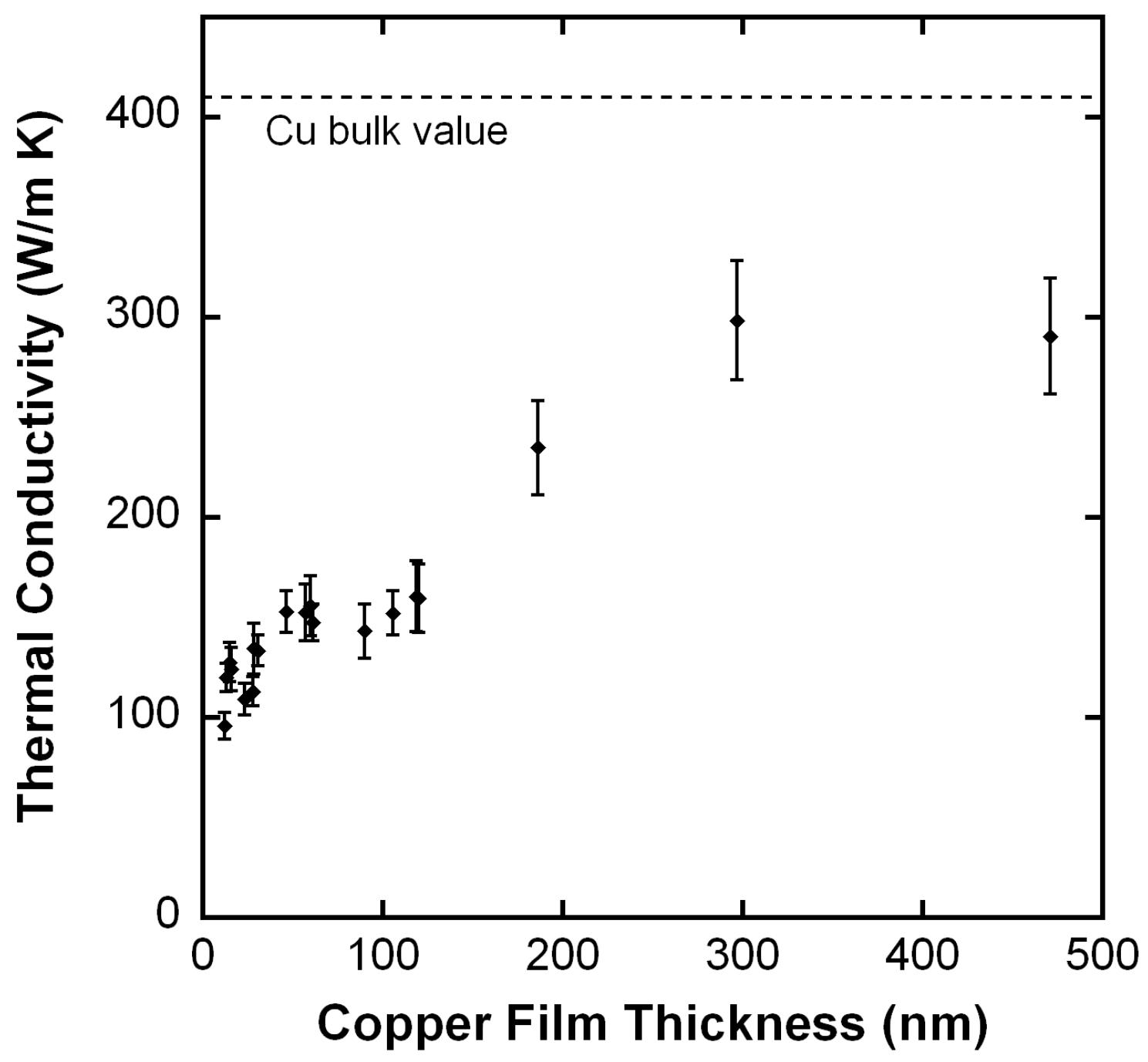

Figure 17: Calculated thermal conductivity of thin copper films from measured thermal parameters, with $k_{S i N}=3.2 \mathrm{~W} / \mathrm{m} \mathrm{K}$ and $\left(\rho c_{P}\right)_{S i N}=2.1 \mathrm{MJ} / \mathrm{m}^{3} \mathrm{~K}$ [23]. 


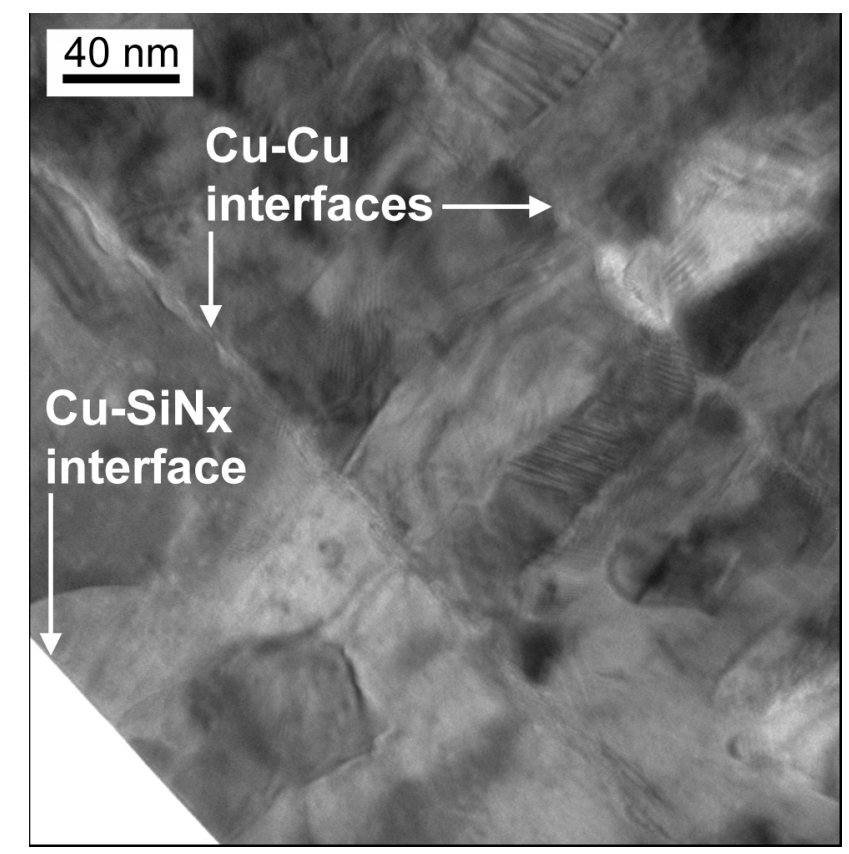

Figure 18: TEM cross-section of a 3-layer $\mathrm{Cu}$ film showing scattering interface. 


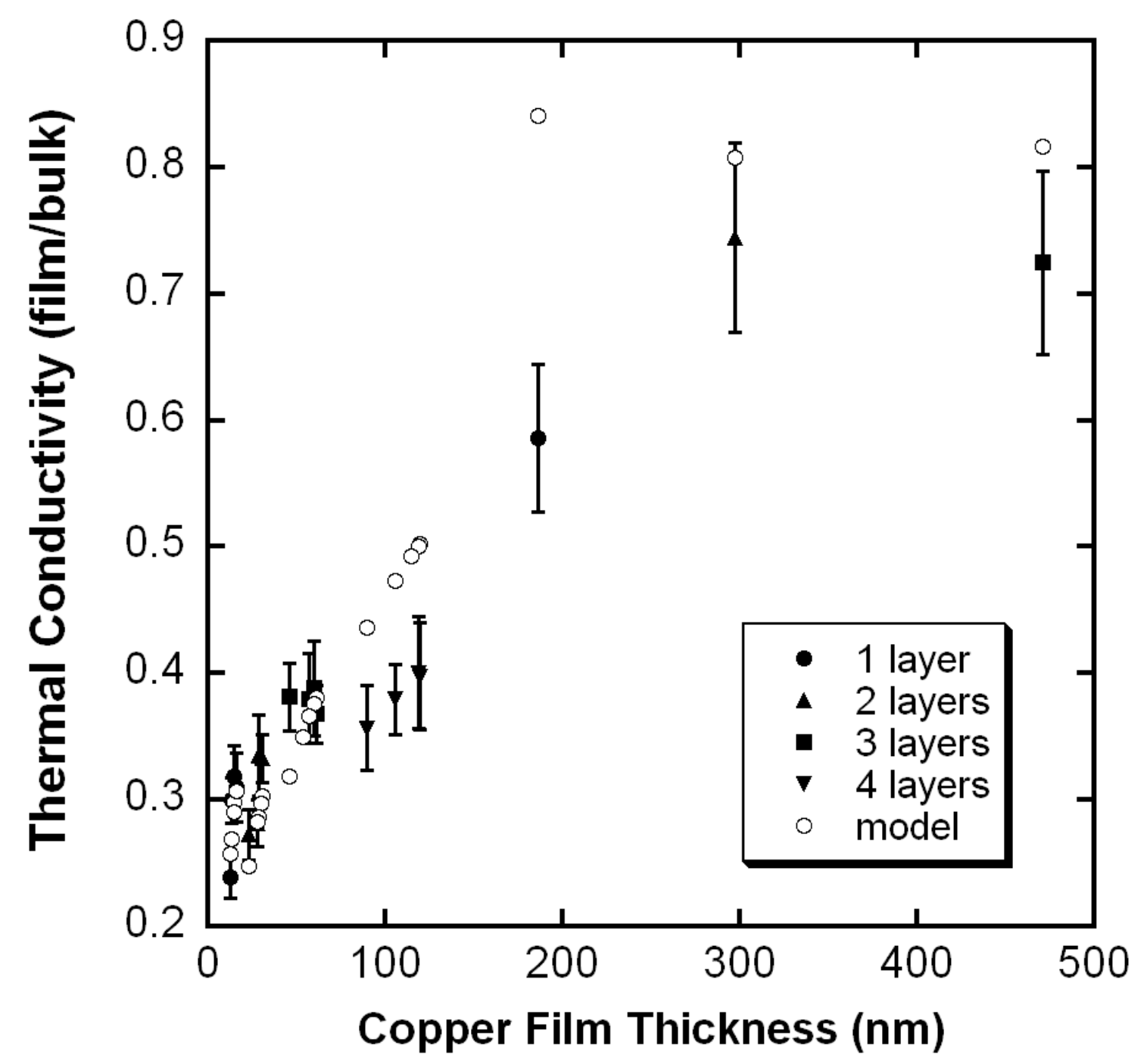

Figure 19: Normalized thermal conductivity measurements of thin copper multilayer films, compared to Qiu's thermal conductivity model results for multilayer copper films. 


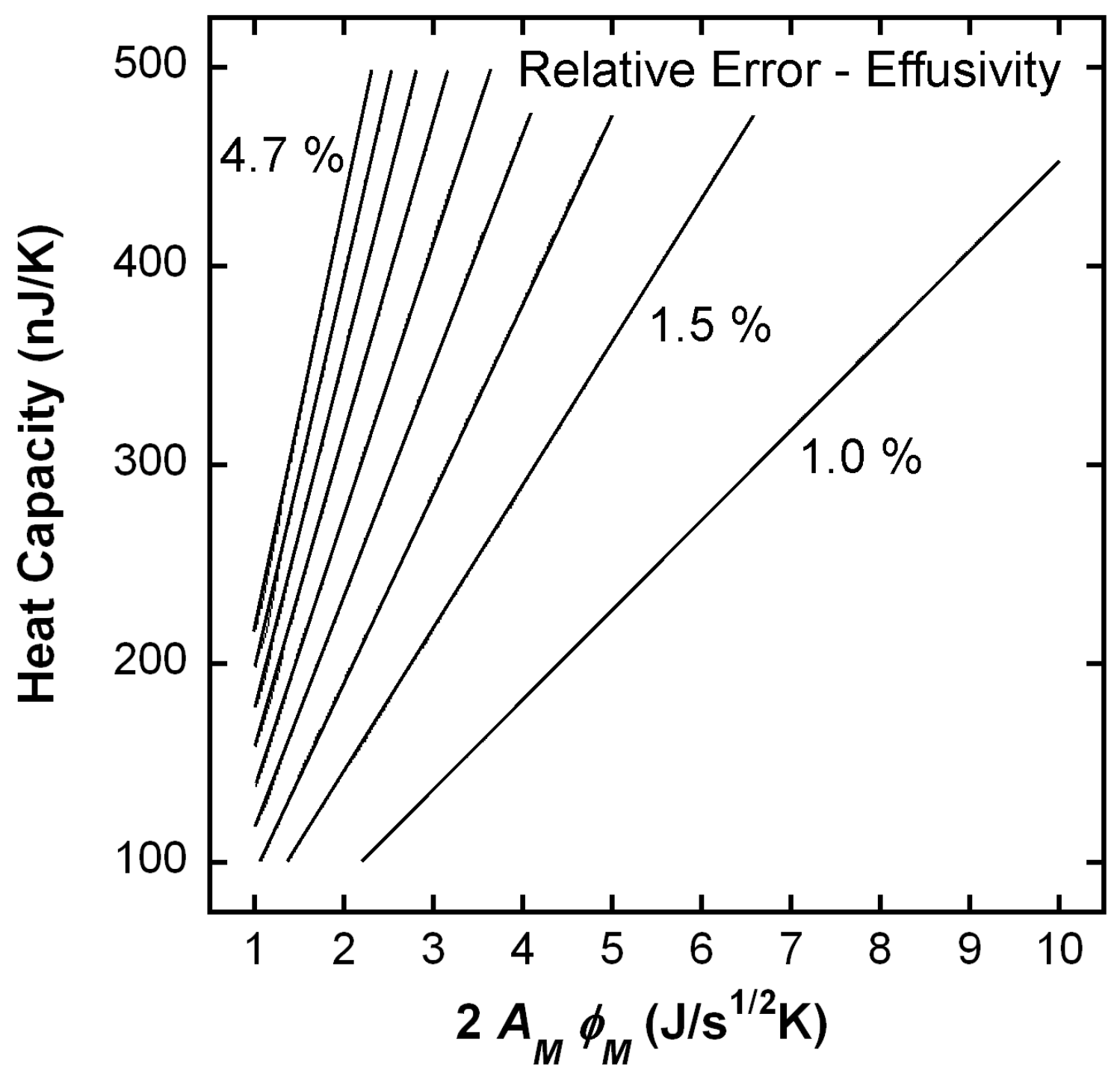

(a) 


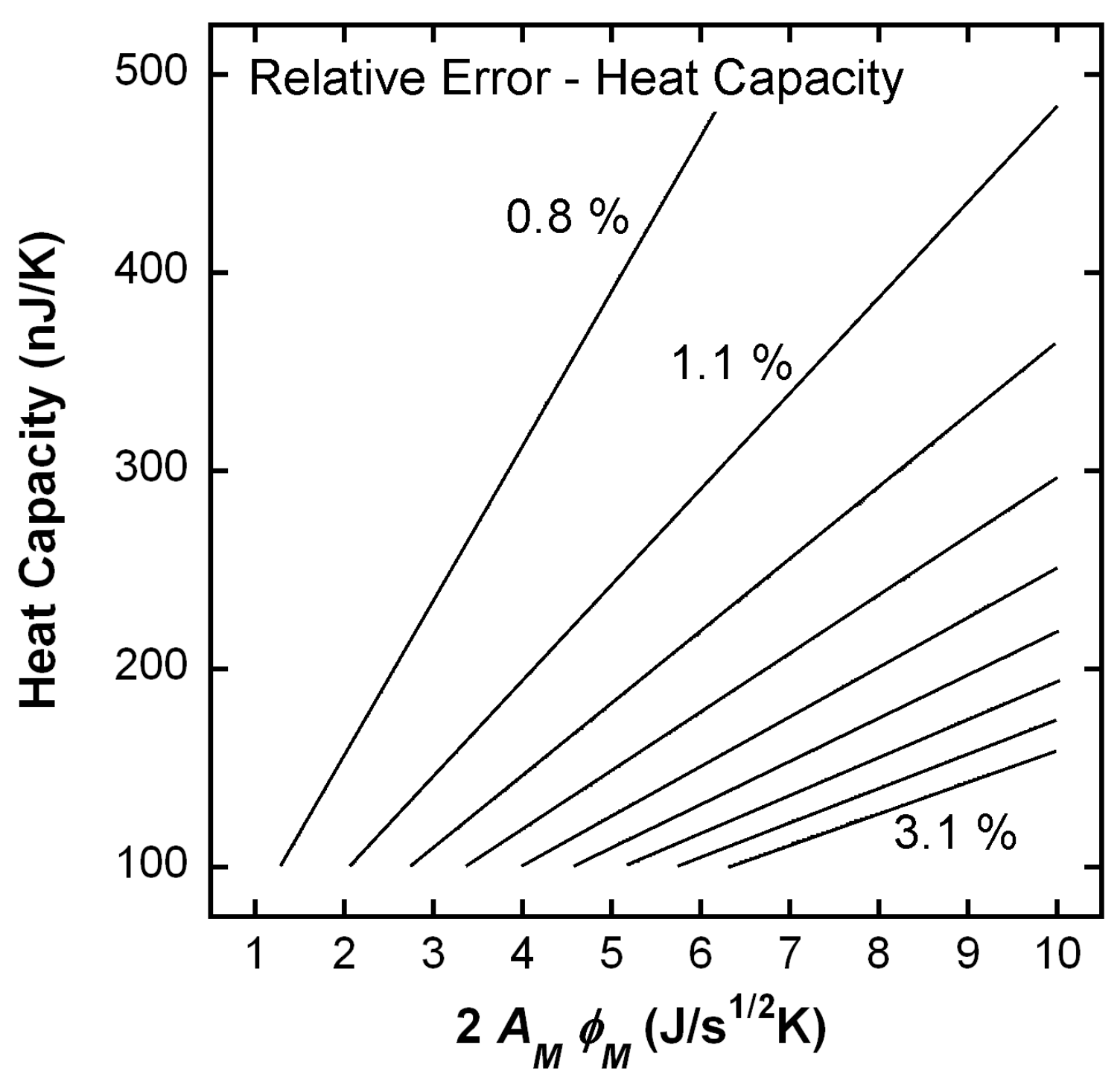

(b)

Figure B1: Relative errors of measured thermal parameters as a function of the value of these thermal parameters, (a) relative error of effusivity and (b) relative error of heat capacity. Data reduction used a moving $0.3 \mathrm{~ms}$ time-span for the heating rate fit and a 4 $\mathrm{ms}$ time-span to fit a $5^{\text {th }}$ order polynomial to the temperature history. 